\title{
Tunable interaction potentials and morphology of polymer-nanoparticle blends
}

Marta Pasquini ${ }^{1}$ and Guido Raos ${ }^{1,}$ a)

Dept. of Chemistry, Materials and Chemical Engineering "G. Natta",

Politecnico di Milano,

via L. Mancinelli 7, 20131 Milano, Italy

We present the results of molecular dynamics simulations of a family of polymer nanocomposite systems. The polymer is described by a generic bead-and-spring model, while the polymer chains and the nanoparticles interact by Hamaker-style potentials. The potential describing nanoparticle-nanoparticle interactions is modified by a tuning parameter $f$, which can be changed continuously between $f=0$ (for fully developed van der Waals attractions between the nanoparticles) and $f=1$ (for completely repulsive interparticle interactions). We explore systematically the effect of the $f$ parameter on the blend morphologies, for two representative nanoparticle sizes. When the polymer-nanoparticle attractions are decreased, the systems undergo a transition from dispersed to aggregated morphologies. The sharpness of the transition gradually increases with the interparticle attractions (i.e., decreasing $f$ ).

a)Electronic mail: guido.raos@ polimi.it 


\section{INTRODUCTION}

Polymer nanocomposites (PNCs) have attracted a great deal of interest from both academia and industry. The dispersion of nanoparticles (NPs) within a polymer matrix can have a profound effect on their properties, making these materials suitable for a wide range of applications, from reinforced or self-healing structural materials to solar cells and stimuli-responsive materials. ${ }^{1,2}$ The physical properties of PNCs are closely related to the nano-scale effects arising from the presence of the nanoparticles, which depend in turn on the overall morphology—particle size and shape, volume fraction and degree of aggregation - and on the modified physical properties of the interfacial polymer layers. ${ }^{3-5}$ Due to the complex interplay of these features, the design of polymer nanocomposites with tailored properties is still an important and open challenge. More generally, understanding the interaction between NPs and (bio)polymers is of primary interest also for biology, nanomedicine and environmental science. ${ }^{6-8}$

The degree of NP dispersion within a polymer matrix is a critical variable, which must be controlled by either thermodynamic or kinetic means (i.e., processing conditions) in order to optimize the final characteristics. ${ }^{9}$ One common strategy to improve the miscibility of the particles with the polymer is to coat them with surfactants or graft polymer-like chains to their surface, in order to avoid or limit their aggregation. ${ }^{10}$ With the right type and degree of functionalization, the net interaction between the nanoparticles (potential of mean force, PMF) can actually be turned from attractive to purely repulsive, driving the system from an aggregated to a well dispersed morphology. ${ }^{11}$

Theory and simulations can play a major role in establishing the connection between the molecular-level interactions, the morphology and the overall properties of a nanocomposite. ${ }^{12-14}$ Fully atomistic models can be fairly realistic, as they are based on increasingly accurate and flexible force fields, including reactive ones. ${ }^{15,16}$ However, they can be computationally very expensive and, above all, they do not always yield the desired qualitative insights into the relationship between interactions, morphology and final properties of the nanocomposites. Typically, they can be used to compute the properties of the polymer shell surrounding one particle and the PMF between a pair of naked or coated particles. ${ }^{17,18}$ Many-body effective interactions involving three or four nanoparticles can also be significant. ${ }^{19,20}$ These potentials could then be used in more coarse-grained models, treating the polymer as an implicit medium. When the aim is to obtain qualitative insights, it is generally more useful to adopt from the outset a coarse-grained model for 
the nanocomposites, exploring the effect of a limited number of adjustable parameters to obtain an overall view of their properties. Thanks to their simplicity, they are also amenable to theoretical analyses, such as those based on the polymer reference interaction site model ${ }^{21,22}$, classical density functional theory ${ }^{23,24}$, and mode-coupling theory for dynamical properties. ${ }^{25,26}$

Most coarse-grained simulations of polymer-NP mixtures are based on a bead-and-spring model for the polymer. ${ }^{27}$ This relies on the well-known Lennard-Jones (LJ) potential ${ }^{28,29}$ for the non-bonded interactions between the polymer beads:

$$
U_{L J}(r)=4 \varepsilon\left[\left(\frac{\sigma}{r}\right)^{12}-\left(\frac{\sigma}{r}\right)^{6}\right]
$$

where $\varepsilon$ represents an attractive well depth and $\sigma$ an effective diameter of the atoms. Several options have been adopted for the NP's. The simplest one is to retain a single LJ site for a whole NP, but using a larger $\sigma .{ }^{30}$ However, this makes sense only for very small particles. A large NP can also be modelled as a rigid aggregate of several LJ sites. ${ }^{31,32}$ This is more realistic but may increase the computational cost, by an extent depending on the particle size and the algorithms for the evaluation of non-bonded interactions. Alternatively, an empirical generalization of the LJ potential allows the introduction of a hard-core diameter $\Delta$ (using different $\Delta$ 's for polymer-particle and particle-particle interactions): $33-36$

$$
U(r)=4 \varepsilon\left[\left(\frac{\sigma}{r-\Delta}\right)^{12}-\left(\frac{\sigma}{r-\Delta}\right)^{6}\right]
$$

This type of potential is very economical from computational point of view, and it can be applied also to relatively large particles $(\Delta \gg \sigma)$. However, it is not "theoretically grounded", so that for example there is no well-defined connection between the particle size, the energy parameter and the range of the potential. Such a connection exists in the Hamaker model.$^{37}$ Here a large object is imagined to be composed of many LJ interaction sites and the overall potential acting between two of them is obtained from the integral of such LJ interactions over their volumes. ${ }^{11,38}$ The total interaction potential if virtually indistinguishable from that obtained from the sum of individual atom-atom interactions, but there is a subtle difference. The particles are perfectly spherical and have no surface roughness, so that they can rotate without experiencing friction. This can have importance consequences for their dynamics, but does not affect their aggregation behavior. We shall provide further details on this model in the next section.

Our aim is to study the phase behavior and morphology of model PNCs by changing systematically the NP-NP and polymer-NP interaction potentials. In doing so, we build on previous work 

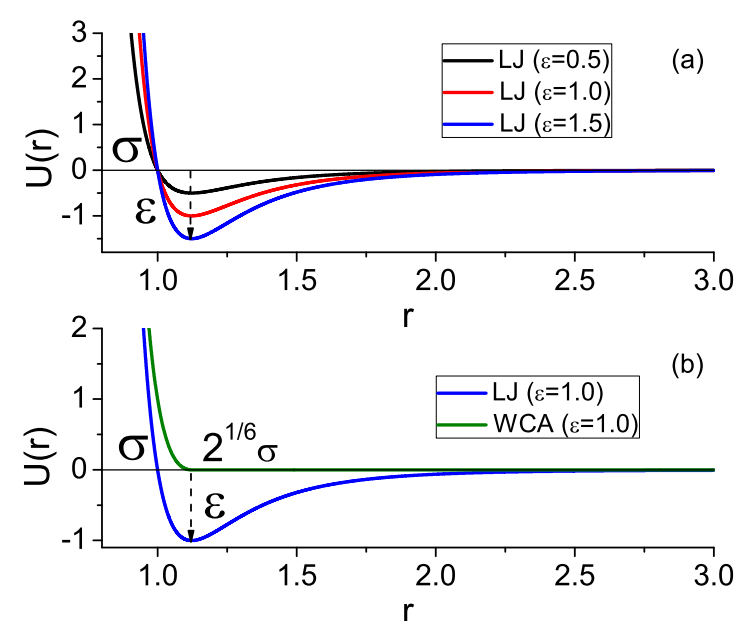

FIG. 1. Panel (a): plot of various LJ potentials with different energy scale parameters and same length scale $(\sigma=1.0)$. Panel (b): plot of LJ and WCA potentials having the same $\varepsilon=1.0$ and $\sigma=1.0$ parameters.

by Meng et al., ${ }^{39}$ who considered systems with purely repulsive interparticle interactions and variable polymer-NP attractions. Their model is justified by the assumption that the NPs have been coated with short surfactants, effectively turning their PMF from attractive to repulsive. ${ }^{40}$ They constructed their NP-NP potential from the Hamaker one, eliminating any attraction by truncating and upward-shifting it at its minimum. This type of strategy was first adopted in the WeeksChandler-Andersen (WCA) modification of the LJ potential, where $r_{\text {cut }}=2^{1 / 6} \sigma$ (panel (b) of Figure 1). ${ }^{41}$ The truncation at the minimum completely eliminates the attractive tail of the potential, without affecting its repulsive part. Repulsive force are known to be especially important for the structure of liquid matter. ${ }^{28,42}$ Unfortunately, one cannot use the same strategy to simulate intermediate situations, between the purely repulsive case and the fully attractive one with a large cutoff ( $\left.r_{\text {cut }} \geq 2.5 \sigma\right)$. Choosing an intermediate value of the cutoff ( $r_{\text {cut }}=1.8 \sigma$, for example) produces a potential with a sharp discontinuity in the force at the cutoff. This could be eliminated by various potential smoothing and interpolation methods, ${ }^{29}$ many of which are implemented in LAMMPS. ${ }^{43}$ These change the net interaction energy but, given the large starting discontinuity, they also produce a significant distortion to the overall shape of the potential, with possible unintended effects on the phase behavior. ${ }^{44}$ Gradually changing the $\varepsilon$ parameter while keeping $r_{\text {cut }}$ at a large value is also not viable as a strategy, because it affects both the attractive and repulsive parts of the potential (panel (a) of Figure 1).

In this work we propose and illustrate an alternative strategy, which allows to continuously tune the attractive part of the interaction potential between two particles, while leaving the repulsive 
one unchanged. We apply it to the Hamaker potential for NP-NP interactions, but the strategy is general and could be applied to other potentials, including the original LJ one and the modified one of Eq. (2). We use this model potential to simulate the bulk phase behavior of the NPs in a polymer melt and to investigate how the NP-NP and the monomer-NP interaction strengths affect the morphology of the composite. Although we do not provide a rigorous theoretical justification, the general idea is that our tunable potential could be used to model nanocomposites in which the functionalization of the NPs with polymer chains or surfactants is only partly effective in compensating the van der Waals attractions. ${ }^{11}$

The following section provides details about our model and simulation protocols. The subsequent ones present our results for representative systems, with two different particle sizes. Conclusions follow.

\section{MODELS AND METHODS}

We have performed molecular dynamics (MD) simulations with the massively parallel, free and open-source code LAMMPS. ${ }^{43}$ In the following we use a notation coherent with the one given in the LAMMPS manual (at https://lammps.sandia.gov), with minor modifications. The polymer chains are represented with the Kremer-Grest bead-and-spring model ${ }^{27}$, which has been extensively used to simulate polymer melts, networks, composites, confined films and solutions. ${ }^{45,46}$ Nanoparticles are perfect spheres of nominal radius $R$, conceptually composed of LJ sites identical to those of the polymer and number density $\rho=1.0 / \sigma^{3}$, with a overall mass $m_{P}=4 \pi \rho m R^{3} / 3$.

The interactions among the chains are described by a truncated and shifted LJ potential:

$$
U_{m m}(r)= \begin{cases}\frac{A_{m m}}{36}\left[\left(\frac{\sigma}{r}\right)^{12}-\left(\frac{\sigma}{r}\right)^{6}\right]-U_{\text {shift }} & : r<r_{c m} \\ 0 & : r \geq r_{c m}\end{cases}
$$

where $A_{m m}$ is a Hamaker constant, which we set equal to $144 \varepsilon$. The " $m$ " in the subscripts stands for monomer. Comparing with Eq.(1), we see that the effective well depth for these interactions is indeed equal to $\varepsilon$ (except for a minor correction arising from the energy shift). The "shift" term ensures that the potential is continuous at the cutoff $r_{c m}=3.0 \sigma$. In addition, contiguous beads along a chain are bonded through a Finitely Extensible Nonlinear Elastic (FENE) potential, with 
a spring constant $k=30.0 \varepsilon / \sigma^{2}$ and a maximum extensibility $R_{0}=1.5 \sigma:^{27}$

$$
U_{F E N E}(r)=-\frac{k R_{0}^{2}}{2} \ln \left[1-\left(\frac{r}{R_{0}}\right)^{2}\right] .
$$

According to the Hamaker model, ${ }^{37}$ which was re-derived by Everaers and Ejtehadi for the more general case of ellipsoidal particles, ${ }^{38}$ the overall potential between two NPs can be obtained by integrating the LJ interactions acting among the sites having a uniform number density $\rho_{i}(\mathbf{r})$ within their volume:

$$
U(r)=\int_{N P_{1}} \int_{N P_{2}} \rho_{1}(\mathbf{r}) \rho_{2}\left(\mathbf{r}^{\prime}\right) U_{L J}\left(\left|\mathbf{r}-\mathbf{r}^{\prime}\right|\right) d V d V^{\prime} .
$$

The exact solution of Eq. (5) leads to a potential composed of an attractive and a repulsive contribution:

$$
U(r)=U_{A}(r)+U_{R}(r) .
$$

These respectively arise from the $(\sigma / r)^{6}$ and $(\sigma / r)^{12}$ parts of the LJ potential in Eq.(1) and are given by:

$$
\begin{aligned}
U_{A}(r)= & \frac{A_{P P}}{6}\left[\frac{2 R_{1} R_{2}}{r^{2}-\left(2 R_{1}+R_{2}\right)^{2}}+\frac{2 R_{1} R_{2}}{r^{2}-\left(R_{1}-R_{2}\right)^{2}}\right. \\
& \left.+\ln \left(\frac{r^{2}-\left(R_{1}+R_{2}\right)^{2}}{r^{2}-\left(R_{1}-R_{2}\right)^{2}}\right)\right]
\end{aligned}
$$

and

$$
\begin{aligned}
U_{R}(r)= & \frac{A_{P P}}{37800} \frac{\sigma^{6}}{r}\left[\frac{r^{2}-7 r\left(R_{1}+R_{2}\right)+6\left(R_{1}^{2}+7 R_{1} R_{2}+R_{2}^{2}\right)}{\left(r-R_{1}-R_{2}\right)^{7}}\right. \\
& +\frac{r^{2}-7 r\left(R_{1}+R_{2}\right)+6\left(R_{1}^{2}+7 R_{1} R_{2}+R_{2}^{2}\right)}{\left(r+R_{1}+R_{2}\right)^{7}} \\
& -\frac{r^{2}-7 r\left(R_{1}+R_{2}\right)+6\left(R_{1}^{2}+7 R_{1} R_{2}+R_{2}^{2}\right)}{\left(r+R_{1}-R_{2}\right)^{7}} \\
& \left.-\frac{r^{2}-7 r\left(R_{1}+R_{2}\right)+6\left(R_{1}^{2}+7 R_{1} R_{2}+R_{2}^{2}\right)}{\left(r-R_{1}+R_{2}\right)^{7}}\right]
\end{aligned}
$$

In equations (7) and (8) $A_{P P}$ is the Hamaker constant for the NP-NP interaction, $R_{1}$ and $R_{2}$ are nominal radii of the two considered particles. The " $P$ " in the subscripts stands for $P$ article. If the beads making up the NPs are assumed to be identical to those of the polymer, then $A_{P P}=4 \pi^{2} \varepsilon=$ $39.478 \varepsilon$. This is the value adopted in our simulations. 


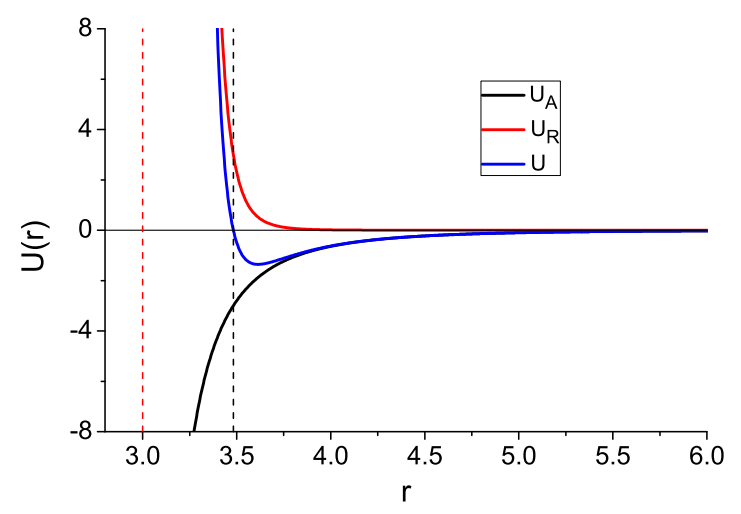

FIG. 2. Total interaction potential between two NPs as function of their distance $r$ (blue line) and its attractive (black) and repulsive (red) components. The red dashed line represents the sum of the nominal radii of the particles $\left(R_{1}=R_{2}=1.5\right)$, while the black dashed line represents their effective diameter $\sigma_{P}$. Distances are measured in units of $\sigma$, energies in units of $\varepsilon$.

The plots of the two terms and of the total potential are shown in Fig. 2. The plot corresponds to the interaction of two NPs with a nominal radius $R_{1}=R_{2}=1.5 \sigma$. The red dashed line in Fig. 2 represents the sum of these nominal radii, while the black dashed line marks the distance at which the total potential is zero. In analogy with the common interpretation of the $\sigma$ parameter of the LJ potential, we shall denote it by $\sigma_{P}$ and consider it as the effective diameter of the NPs. The effective (as opposed to the nominal) radius of a NP is equal to half of this. Note that the effective radius is appreciably larger than the nominal one ( $1.74 \sigma$ versus $1.50 \sigma$, in this case).

The total interaction potential of Eq.(6) is derived from a non-truncated LJ interaction potential of Eq.(1). From an operational point of view, in order to establish a cutoff to be used in LAMMPS, it must also be truncated and shifted, in analogy with Eq.(3). Let us define its range as the distance at which the attractive NP-NP interactions decay to a sufficiently small value. For the two cases considered in this study, with nominal particle radii of $1.5 \sigma$ and $2.5 \sigma$, this occurs at $6.0 \sigma$ and $10.0 \sigma$. We shall use these as the outer cutoff of the model ( $r_{\text {att }}$ in the following).

The cutoff for NP-NP interactions determines the range of the interaction, but it can also be used to transform an attractive potential into a repulsive one, following the logic of the WCA truncation. ${ }^{39,41}$ As mentioned in the Introduction, here we present a simple method to tune the nanoparticles' interaction potential in a continuous way, from completely repulsive to attractive. The potential that we use is a weighted linear combination of two limiting cases: $U(r)$ taken with an outer cutoff $r_{\text {att }}$ that determines an attractive interaction on one hand, and the same $U(r)$ taken 
with an inner cutoff $r_{\text {rep }}$ giving rise to a repulsive potential on the other. The two limiting cases read as:

$$
\begin{aligned}
& U_{\text {rep }}(r)= \begin{cases}U(r)-U\left(r_{r e p}\right), & \text { for } r<r_{r e p} \\
0, & \text { for } r>r_{r e p}\end{cases} \\
& U_{\text {att }}(r)= \begin{cases}U(r)-U\left(r_{\text {att }}\right), & \text { for } r<r_{a t t} \\
0, & \text { for } r>r_{\text {att }}\end{cases}
\end{aligned}
$$

where $U(r)$ is the untruncated potential of Eq. (6). The full potential $U_{m i x}(r)$ can be expressed in terms of a mixing parameter $f$ determining the weight of $U_{r e p}$ and $U_{a t t}$ in the final potential:

$$
U_{\text {mix }}(r)=f U_{\text {rep }}(r)+(1-f) U_{\text {att }}(r)= \begin{cases}U(r)-(1-f) U\left(r_{\text {att }}\right)-f U\left(r_{\text {rep }}\right), & \text { for } r<r_{\text {rep }} \\ (1-f) U(r)-(1-f) U\left(r_{\text {att }}\right), & \text { for } r_{\text {rep }} \leq r<r_{\text {att }} \\ 0, & \text { for } r>r_{\text {att }}\end{cases}
$$

Thus, for $f=1.00$, the potential is completely repulsive, while for $f=0.00$ the interaction includes the full attractive part. In Fig. 3 the potentials $U_{m i x}(r)$ are plotted for both the small and large NPs, with $R=1.5 \sigma(f=0.00,0.25,0.50,0.75$ and 1.00$)$ and $R=2.5 \sigma(f=0.00,0.50$ and 1.00$)$.
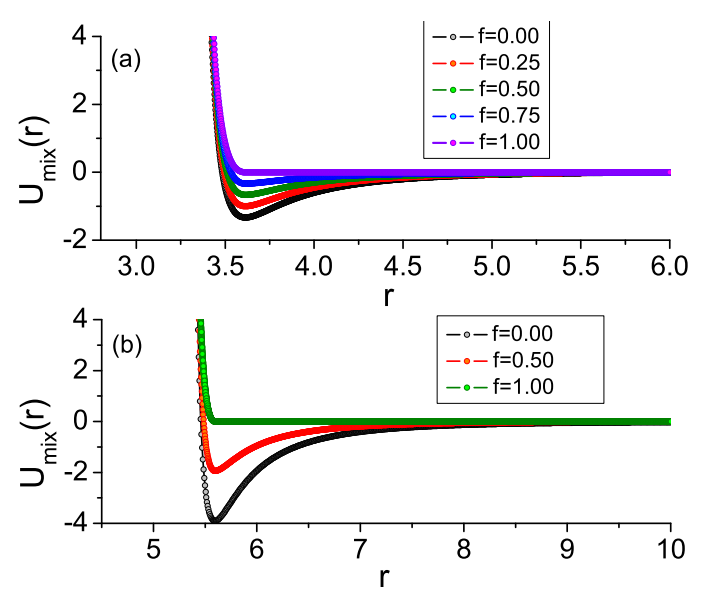

FIG. 3. NP-NP interaction potentials $U_{m i x}$ for NPs with nominal radius $R=1.5$ (panel (a)) and $R=2.5$ (panel (b)), for the mixing parameters $f$ considered in this work. Distances are measured in units of $\sigma$, energies in units of $\varepsilon$.

The interaction between monomers and NPs is derived from the particle-particle interaction 
and the potential has the following expression

$$
\begin{aligned}
U_{P m}(r)= & \frac{2 R^{3} \sigma^{3} A_{P m}}{9\left(R^{2}-r^{2}\right)^{3}} \times \\
& {\left[1-\frac{\left(5 R^{6}+45 R^{4} r^{2}+63 R^{2} r^{4}+15 r^{6}\right) \sigma^{6}}{15(R-r)^{6}(R+r)^{6}}\right] . }
\end{aligned}
$$

In the above equation $A_{P m}$ is the Hamaker constant determining the interaction strength between NPs and monomers. Following Meng et al. ${ }^{39}$ we employ $A_{P m}$ as an adjustable parameter, to drive the system from dispersed to aggregated morphologies.

As we have already mentioned, we presents results for systems with two particle sizes. The polymer chains are always composed of 24 beads. It has been proved that the chain length is not a crucial element when the chains have more than 10 monomers, as long as one is concerned with the blend morphologies. ${ }^{39}$ Of course, the polymer chain length has a strong effect on dynamical properties (e.g., viscosity and diffusion), but these are not the main concern of this paper. The first set of simulations (system $\mathrm{S}$ ) contains 220 small NPs with a nominal radius of $R=1.5 \sigma$, which corresponds to an effective radius of 1.74 and an effective diameter $\sigma_{P}=3.48 \sigma$. The NPs are dispersed in a matrix composed of 2640 chains. Since the effective volume of a particle is $V_{P}=22.108 \sigma^{3}$ and the equilibrated box volume is about $78000 \sigma^{3}$, the particle volume fraction is $\phi \simeq 6.2 \%$. In the second set of simulations (system L) there are larger NPs, with a nominal radius equal to $2.5 \sigma$ and an effective diameter $\sigma_{P}=5.46 \sigma$. The box contains 8000 chains, for a total of 192000 beads and 128 NPs. In this case the volume fraction is $\phi \simeq 4.8 \%$.

The potential energy functions and the associated forces were pre-computed and written to files as numerical tables, in a LAMMPS-compatible format. The Python code for this task is available as supplementary information to this article.

All simulations were performed with periodic boundary conditions in all directions. The initial systems were generated as boxes containing the desired number of polymer chains and NPs, the latter initially having $R=0$ and then gradually increased to the target size. A soft potential was initially employed to eliminate overlaps. Subsequently, the soft potential was replaced by the purely repulsive one $(f=1)$, and these equilibrated structures were used as a starting point for the simulations with interparticle attractions $(f<1)$. The production runs were performed for each set of parameters under isothermal-isobaric conditions (NPT ensemble) at temperature $k_{B} T=\varepsilon$ and pressure $P=0.0 \varepsilon / \sigma^{3}$ with a timestep of $0.005 \tau$, where $\tau=\sigma(m / \varepsilon)^{1 / 2}$, and for a total of $7 \cdot 10^{6}-2 \cdot 10^{7}$ timesteps, depending on the interactions and on the particle size. The systems were 

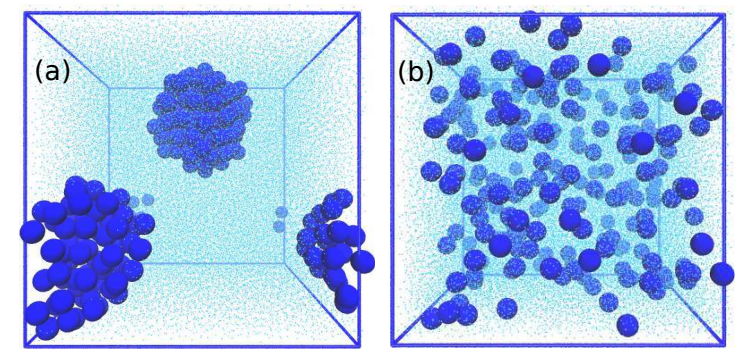

FIG. 4. Snapshots from simulations of system $\mathrm{S}$ and $f=0.00$. In panel (a) the system is characterized by $A_{P m}=105$, while in panel (b) the system is characterized by $A_{P m}=135$.

considered equilibrated when the density, the non-bonded interaction energy and the NP-NP Radial Distribution Function (RDF) became steady. The root-mean-square displacements (RMSDs) of the NPs and of the polymers' center-of-mass were also monitored, to ensure that they reached values larger that the side of the simulation box.

\section{RESULTS}

Following common practice, in the following plots and the associated discussion we switch to a system of reduced units based on the LJ potential. ${ }^{29}$ Lengths are measured in units of $\sigma$, energies in units of $\varepsilon$, masses in units of the monomer mass $m$, times in units of $\tau$, etc.. As a results, all quantities effectively become adimensional, and we report them as such.

\section{A. System S}

\section{Phase diagram}

Typical snapshots from our simulations are shown in Fig. 4 for two S-type systems. Panel (a) illustrates a situation with unfavourable polymer-nanoparticle interactions, with a Hamaker constant $A_{P m}=105$. Some aggregation between NPs occurs regardless of the NP-NP interaction potential, i.e. regardless of the mixing parameter $f$. The system in panel (b) of Fig. 4 is characterized by very favourable polymer-nanoparticle interactions with $A_{P m}=135$. In this case the NPs are clearly well dispersed, despite of their strong attractive interactions $(f=0)$.

Panel (a) of Fig. 5 shows some representative plots of nanoparticle-nanoparticle RDFs, $g_{P P}(r)$. Following Meng et al. ${ }^{39}$, the values of the RDF at the first peak can be used to define the aggre- 


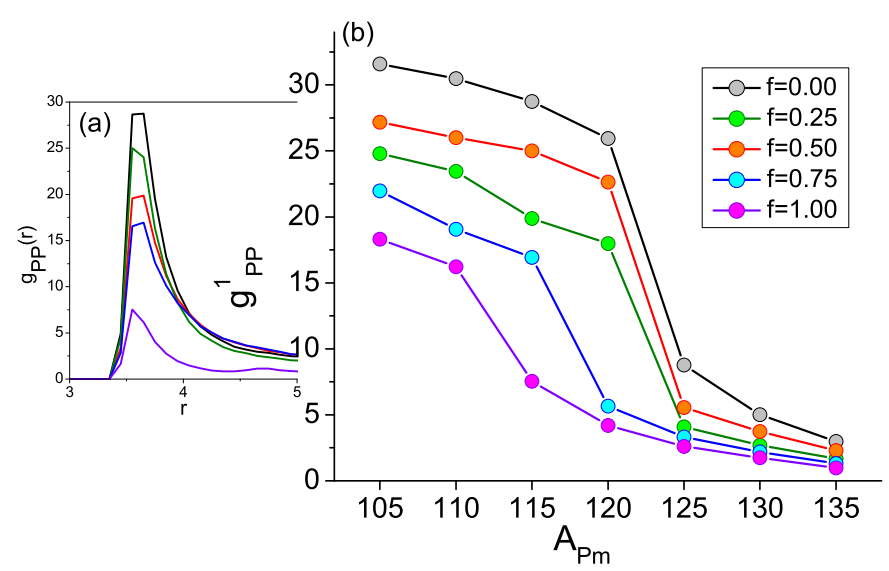

FIG. 5. Panel (a): $g_{P P}(r)$ for different values of the "mixing" parameter for systems of type $\mathrm{S}$ and $A_{P m}=115$. Panel (b): $g_{P P}^{1}$ as function of the Hamaker constant $A_{P m}$.

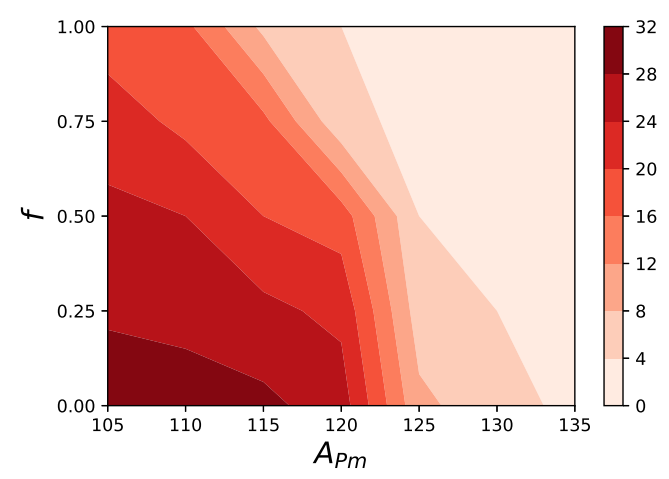

FIG. 6. Phase diagram for systems of type $\mathrm{S}$ with particle volume fraction $\phi \simeq 6.2 \%$, giving $g_{P P}^{1}$ as function of $A_{P m}$ and $f$.

gation state of the NPs and build a phase diagram. We denote these values by $g_{P P}^{1}$. In panel (b) of Fig. 5, we plot $g_{P P}^{1}$ as function of $A_{P m}$ for different values of the $f$ parameter. The same data are plotted as a phase diagram in Fig. 6, where the darker colors indicate more aggregated morphologies.

In general, $g_{P P}^{1}$ decreases as $A_{P m}$ increases, indicating that the system becomes more dispersed. Although this trend is preserved regardless of the interaction between NPs, the mixing parameter $f$ has a strong impact on the aggregation state of the system. The inflection point of the graph, which identifies the transition from aggregation to dispersion, is shifted towards lower $A_{P m}$ values as the 
NPs interaction becomes less attractive. Moreover, the steepness of the curves in the transition region depends on $f$. Strongly attractive potentials $(f=0.00-0.25)$ give rise to sharper transitions than weakly attractive and repulsive ones.

For large values of $\mathrm{A}_{P m}$, a similar level of dispersion is attained for all the considered mixing parameters. In this regime the polymer-NP interaction becomes strong enough to overcome the NP attractions and the systems ends up in a dispersed state, independently of $f$. Instead, when $A_{P m}$ decreases, the interaction between nanoparticles takes an important role in determining their aggregation state, so that the curves seem to reach very different limit values. This observation suggests that the final aggregation state is different in this limit.

In order to further understand the differences between the aggregation states obtained for different NP-NP interactions, we focused on three families of cases, analyzing properties and structures of the nanoparticle clusters. We discussing them in turn, going from the more aggregate to the more dispersed ones $\left(A_{P m}=105,120\right.$ and 135, respectively).

\section{Aggregated state: $A_{P m}=105$}

Panel (a) of Fig. 7 shows the NP-NP radial distribution functions for different values of the mixing parameter. The first peak appears at a distance about twice the effective radius of the NPs, at $r=\sigma_{P}=3.48$, which corresponds to direct contact between nanoparticles. The value at the peak maximum depends strongly on $f$, as we have already pointed out. The peak is rather broad, as it extends to distances $r \simeq 5$, where we find the first minimum in $g_{P P}(r)$. Note that the value at this minimum is almost independent of $f$ and well above unity, indicating again a strong degree of nanoparticle aggregation. A second peak corresponding to $r \simeq 2 \sigma_{P}$ is present for all the $f$ values, although it becomes bimodal in the more attractive cases. $g_{P P}(r)$ tends to unity only at very large distances. Polymer-mediated contacts among the nanoparticles, such as those described by Hooper and Schweizer ${ }^{21}$, would produce a peak in the RDF at $r \simeq 1+\sigma_{P}=4.5$. We do not see a distinct peak, but the RDF is still very large at this distance. Thus, there seems to be a continuum of aggregation types for the nanoparticles, which comprises both direct and polymer-mediated contacts. When the interaction between NPs is purely repulsive $(f=1.00)$, the NPs aggregation is the result of an entropy driven phase separation, in analogy with the depletion interaction that occurs in systems containing larger particles. ${ }^{47}$ Instead, the aggregation becomes increasingly energy-driven when $f$ decreases. 

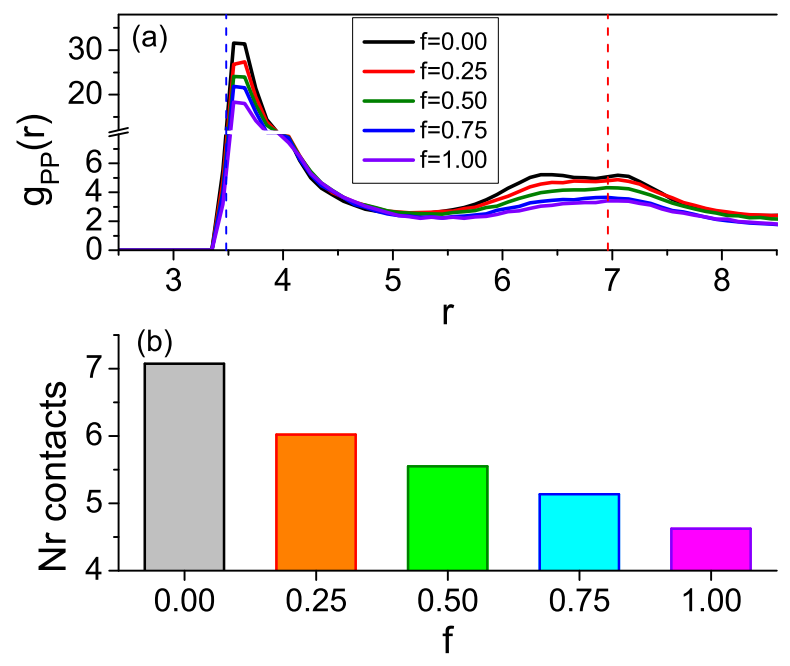

FIG. 7. Panel (a): NP-NP RDF for the considered values of the mixing parameter $f$. The blue and red dashed lines represent, respectively, $\sigma_{P}$ and $2 \sigma_{P}$. Panel (b): average number of NP-NP contacts as function of the mixing parameter. Systems are characterized by $A_{P m}=105$.

The average number of interparticle contacts, also known as the coordination number, can be obtained by counting the average number of NPs within a certain cutoff distance from a reference one. It is also equal to the integral of $4 \pi n_{P} r^{2} g_{P P}(r)$, from zero to the cutoff, where $n_{P}$ is the number density of nanoparticles. The choice of the cutoff is somewhat arbitrary, especially in these cases where the RDF displays a very broad first peak. Since we are interested in highlighting the formation of direct interparticle contacts and exclude the polymer-mediated ones, we have set the cutoff at $r=4.25$. Panel (b) of Figure 7 shows that this number decreases steadily, from 7 to 4.5 as the attractive interactions are progressively switched off.

We also computed the number and size of the clusters formed by the nanoparticles. A cluster is an ensemble of NPs, each of which is within a threshold distance from at least another nanoparticle. For the threshold we use the same value as above, $r=4.25$. Fig. 8 shows the average number of nanoparticles within a cluster. In the fully attractive case with $f=0$, there are only a few clusters contain many NPs. Diffusion of these clusters is very slow but, given sufficient time, they would probably aggregate further to give a complete macrophase separation. Instead, the repulsive system with $f=1$ forms fairly well-defined but relatively small clusters, containing about 15 particles on average. The three systems with intermediate $f$ 's are apparently rather similar, with larger but limited aggregation number. NP aggregation stops at the nanoscale level, in this case.

More information about the local nanocomposite structure can be obtained through the distri- 
Potentials and morphology of polymer-nanoparticle blends

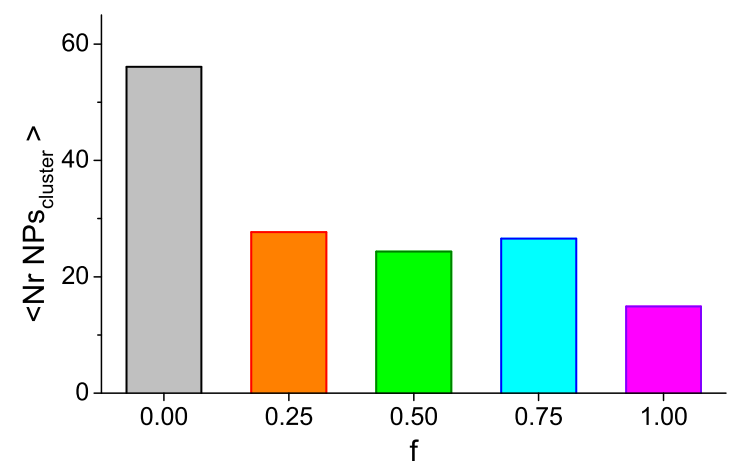

FIG. 8. Average number of NPs within clusters for systems of type $\mathrm{S}$ with $A_{P m}=105$.

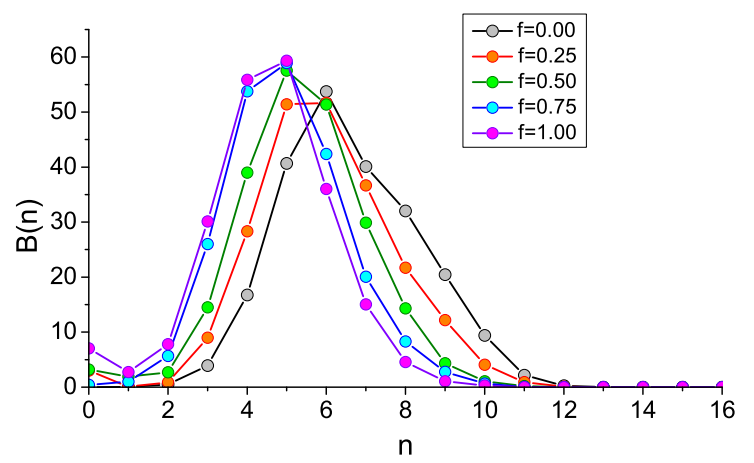

FIG. 9. Distribution of number of nearest neighbors per NP for the considered vales of the mixing parameter $f$, as obtained for systems of type $\mathrm{S}$ with $A_{P m}=105$.

bution of the coordination number around each NP, $B(n)$. The latter is defined as:

$$
B(n)=\frac{\sum_{s=1}^{N_{s}} N_{b}^{s}(n)}{N_{s}}
$$

where $N_{b}^{s}(n)$ is the number of NPs having $n$ neighbors in the considered snapshot and $N_{s}$ is the number of snapshots. Here we averaged over 50 snapshots, taken every 10000 timesteps from an equilibrated dynamics. Fig. 9 shows the effect of the NP-NP interaction on the $B(n)$ distribution. In particular, for more attractive potentials $(f \leq 0.50), B(n)$ has a quite large peak, centered on $n \simeq 6$ and stretching up to $n=10$. When $f$ increases and the NP-NP potential becomes more repulsive, the distribution becomes narrower, with at most $n \simeq 8$ nearest neighbors. 

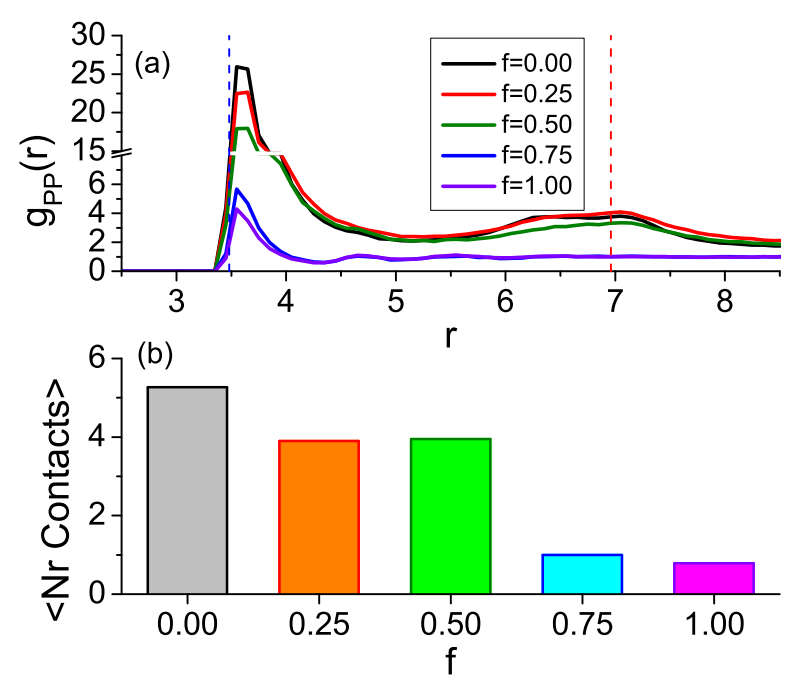

FIG. 10. Panel (a): NP-NP RDFs as obtained for different values of the mixing parameter $f$. The blue and red dashed lines represent, respectively, $\sigma_{P}$ and $2 \sigma_{P}$. Panel (b): average number of NP-NP contacts as function of $f$; the upper limits of the integration are set at 4.25. All the data refer to systems with $A_{P m}=120$.

\section{Intermediate state: $A_{P m}=120$}

Intermediate values of the Hamaker constant for polymer-nanoparticle interactions give rise to aggregated or dispersed systems, depending on the interaction acting between the NPs. Naturally, in these cases it is particularly interesting to investigate the effects of the mixed NP-NP interactions. The NP-NP RDFs (panel (a)) and the average number of NP contacts (panel (b)) are shown in Fig. 10 as function of the mixing parameter $f$, for systems with $A_{P m}=120$.

When $f$ goes from 0.50 to $0.75, g_{P P}(r)$ abruptly changes its shape. We are now in a situation where the final morphology of the system is very sensitive to the details of the interparticle interactions, which depend in turn on the degree and type of NP functionalization. For more attractive NP-NP interactions, the distributions resemble the ones described in the previous sub-section, with a marked first peak at $r \simeq \sigma_{P}$ and long-range oscillations. In the more repulsive cases, the first peak undergoes a sharp drop and other small, distinct peaks appear at $r \simeq \sigma_{P}+1$ and $r \simeq \sigma_{P}+2$, indicating the formation of "polymer-bridged" structures, ${ }^{21}$ where the nanoparticles are separated by one ore two polymer chains. For larger distances, $g_{P P}(r)$ reaches the unit value, describing the loss of correlation between the nanoparticles. The same trend can be observed for the average number of NP contacts (panel (b) of Fig. 10), which takes values of 4-5 for $f \leq 0.50$ and suddenly drops to 1 or less for more repulsive interactions. 


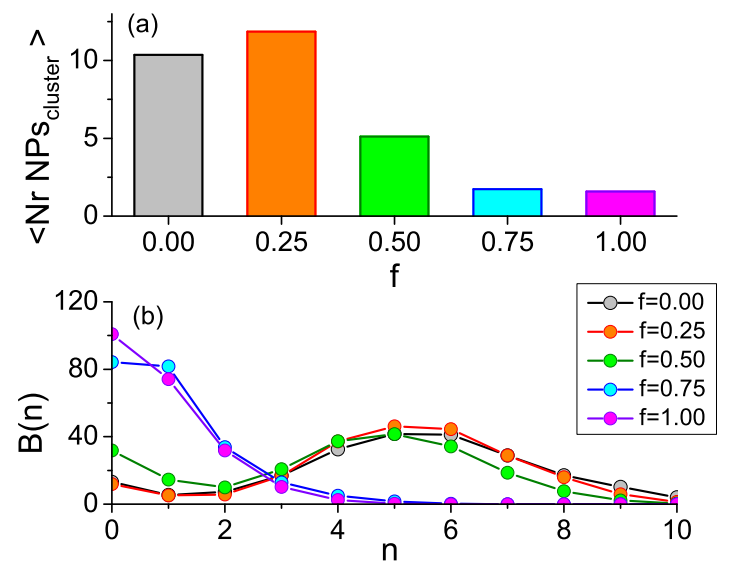

FIG. 11. Panel (a): average number of NPs per cluster. Panel (b): distribution of the average number of nearest neighbors per NP. All the data refer to system of type $\mathrm{S}$ with $A_{P m}=120$.

The average number of NPs within the clusters, shown in panel (a) of Fig. 11 has the same general trend observed for the radial distribution functions, with strong differences between the attractive and repulsive cases. However, from the point of view of this quantity, the transition appears to be more gradual: the system with $f=0.5$ shows intermediate degrees of aggregation. Thus, from the point of view of the cluster size, the transition between the nano-segregated and the dispersed states appears to be more gradual.

The distributions of the nearest neighbors, reported in the (b) panel of Fig. 11, show a peak at $n \simeq 5-6$ in the attractive cases with $f \leq 0.5$. They abruptly shift to much smaller value of $n$ when the potential becomes more repulsive, indicating a very limited clustering of the NPs.

Since the final equilibrium morphology is so sensitive to the tuning of the NP-NP interactions, one might wonder whether these could have some effects also on the dynamical properties of these systems. The simplest of such properties are the diffusion coefficients. It is possible to examine both those of the NPs and of the polymer chains, respectively $D_{N P}$ and $D_{\text {poly }}$. These can be computed from the mean-square displacement (MSD) of their center-of-mass:

$$
D=\lim _{t \rightarrow \infty} \frac{\left\langle\left(\mathbf{X}\left(t+t_{0}\right)-\mathbf{X}\left(t_{0}\right)\right)^{2}\right\rangle}{6 t}
$$

where the angular brackets indicate a double average, over all particles (or all chains) and over all possible time origins $t_{0}$. Rather than taking the limit for $t$ tending to infinity, the diffusion coefficients are commonly obtained by linear fits of the MSDs versus time, as shown in Figure 12 for one representative cases. 


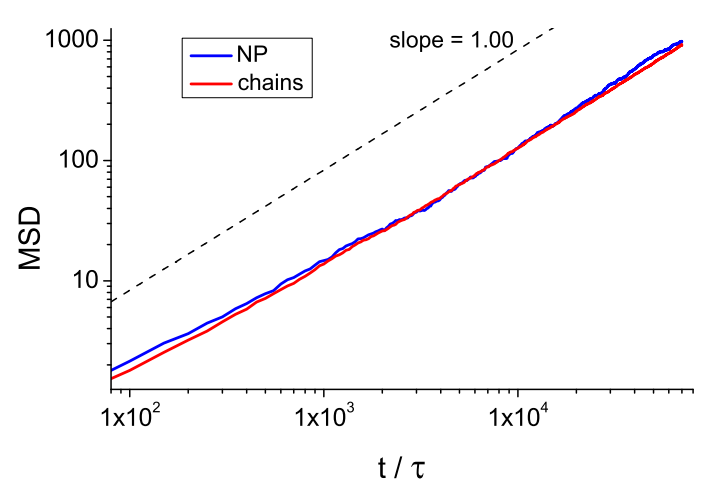

FIG. 12. MSD of the polymer chains and of the NPs, for system S with $A_{P m}=120$ and $f=0.75$ reported in logarithmic scale. The dashed black line indicates the linear behavior, for comparison.

The results for all diffusion coefficients are collected in Fig. 13. In general, the polymer chains have a faster diffusion than NPs, thanks also to their relatively short length. For the Kremer-Grest model, entanglement effect start to be felt for chains of over 100 beads, ${ }^{48}$ i.e. four times larger than our case. The two panels of Fig. 13 show that tuning the NP-NP interactions has a similar effect on the polymer and nanoparticle diffusion coefficients, but in the former case the change is of the order of $10 \%$, to be compared with $100 \%$ or more in the latter. Thus, we concentrate on the discussion of NP diffusion.

On the attractive side, we observe that the NPs in the system with $f=0.25$ are much more mobile than those with $f=0.00$, even though they had a similar morphology (as extracted from the RDFs, see Figs. 10 and 11). The particle mobility increases further on decreasing their attraction, but surprisingly it reaches a maximum at $f=0.75$, rather than in the fully repulsive case with $f=1.00$. This is somewhat surprising and unexpected. We see an analogy with a model for diffusion of polymer chains on random heterogeneous surfaces, where activation energy is also a non-monotonic function of the average polymer-surface interaction. ${ }^{49,50}$ We speculate that the enhancement of diffusive dynamics by weakly attractive NP-NP interactions depends on the ease with which interparticle contacts can be continuously formed and destroyed, leading to continuous reconfigurations of small and highly mobile clusters. This result is not the main focus of our work and should be investigated further, through the simulation of a wider range of systems ${ }^{35}$ and possibly mode-coupling calculations generalizing those of refs. ${ }^{25,26}$ (which focused on the limit of a single nanoparticle, where NP-NP interactions are irrelevant). 

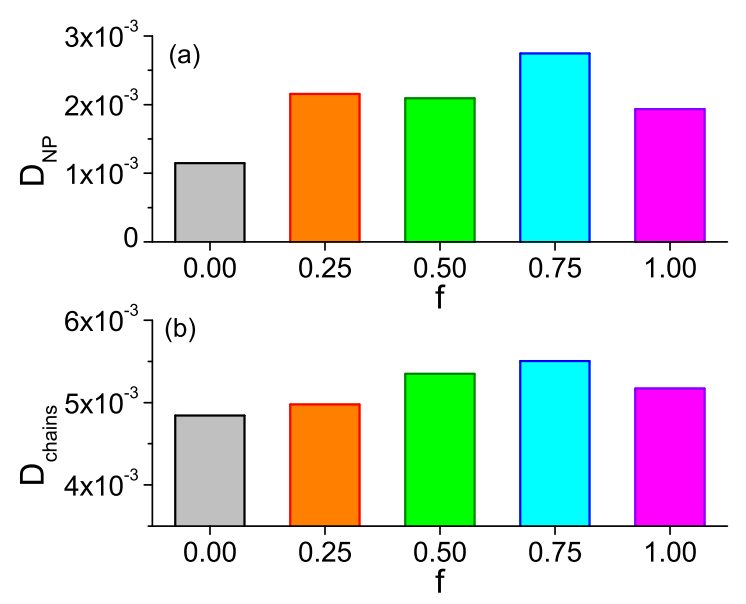

FIG. 13. Diffusion coefficients as obtained from the MSD for system of type $\mathrm{S}$ with $A_{P m}=120$. Panel (a): results for the nanoparticles. Panel (b): results for the polymer chains.

\section{Dispersed state: $A_{P m}=135$}

We finally turn to a situation with strongly favourable interaction between monomer-NP interactionss $\left(A_{P m}=135\right)$. As already anticipated, we expect a fairly well dispersed morphology, regardless the potential acting between NPs. The NP-NP radial distribution functions are reported in the panel (a) of Fig. 14. When $f=0.0$, the RDF shows a relatively small but well-defined peak at about $r=\sigma_{P}$, which decreases further when $f$ increases. Furthermore, there are additional peaks at $r \simeq \sigma_{P}+1$ and $r \simeq \sigma_{P}+2$, as already observed for the more repulsive cases in the previous subsection (Fig. 10). The average number of NP contacts also decreases as $f$ increases (panel (b) of Fig. 14). Even in the most attractive case, this coordination number is only about 0.5 , in agreement with the good degree of dispersion which is observable in Figure 4. Also the average cluster size and the distribution of nearest neighbours (not shown here) indicate little or no aggregation, for all $f$ values.

\section{B. System L}

Two representative snapshots for system L are displayed in Figure 15. Again, we observe a range of situations spanning dispersed and nano-segregated morphologies.

Similarly to what has been done for system S, we classified the possible NP aggregation states on the basis of the value of the first maximum in their RDFs. Panel (a) of Fig. 16 contain their plots for various values of $f$ and $A_{P m}=90$. This corresponds to the intermediate range of polymer- 
Potentials and morphology of polymer-nanoparticle blends
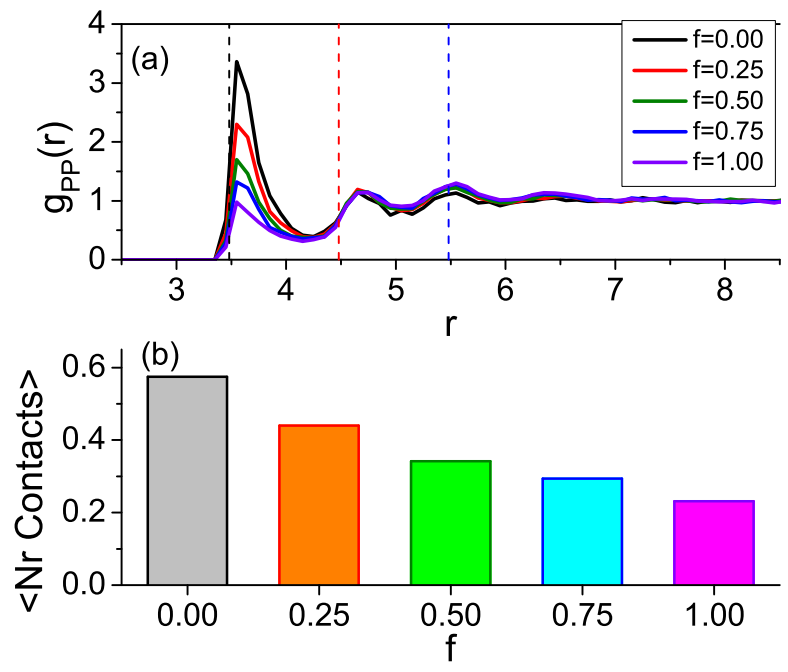

FIG. 14. Panel (a): NP-NP radial distribution functions as obtained for different values of the mixing parameter $f$. The black, red and blue dashed lines represent respectively $\sigma_{P}, \sigma_{P}+1$ and $\sigma_{P}+2$. Panel (b): average number of NP-NP contacts as function of $f$; the upper limit of the integration is 4.25. All data refer to systems of type $\mathrm{S}$ with $A_{P m}=135$.
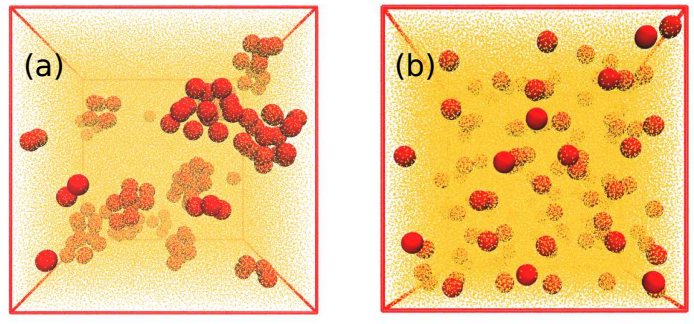

FIG. 15. Snapshots from simulations of system L. In panel (a) the system is characterized by $A_{P m}=70$ and $f=0.00$, while in panel (b) the system is characterized by $A_{P m}=105$ and $f=1.00$.

NP interactions, where the morphology is most sensitive to $f$. Indeed, the dispersion/aggregation transition is found lower values of NP-monomer Hamaker constant $\left(A_{P m}=85-100\right.$, instead of 110-125 in system $S$ ) as shown in panel (b) of Fig. 16. The resulting phase diagram is reported in Fig. 17.

The main trends are similar to those found for system S: the system is less aggregated for higher values of the $A_{P m}$ constant, independently of the mixing parameter $f$. However, this parameter affects both the position of the transition and the steepness of the curves. In general, the progressive inclusion of NP-NP attractions by decreasing $f$ produces a sharper, first-order-like transition be- 
Potentials and morphology of polymer-nanoparticle blends
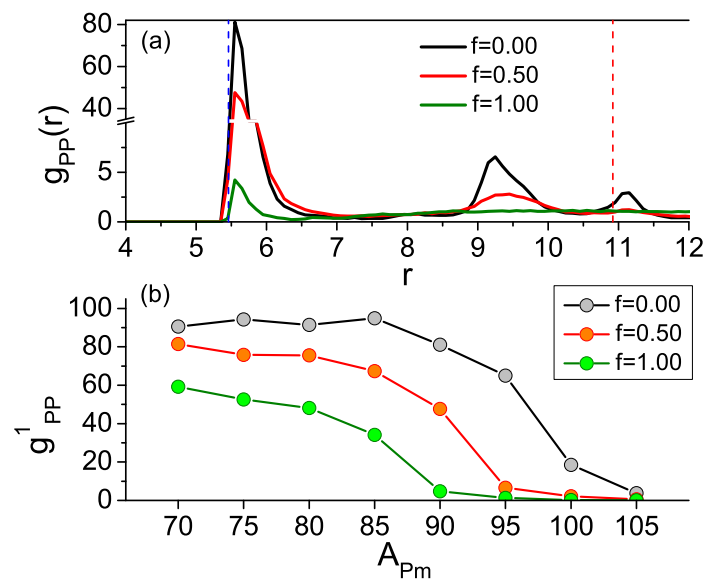

FIG. 16. Panel (a): NP-NP radial distribution functions as obtained for $A_{P m}=90$ and for different values of $f$. The blue and red dashed lines represent, respectively, $\sigma_{P}$ and $2 \sigma_{P}$. Panel (b): value of $g_{P P}(\mathrm{r})$ at its first peak as a function of the Hamaker constant $A_{P m}$. All data refer to systems of type L.

tween aggregated and dispersed morphologies. Very strong interactions between nanoparticles and monomers lead to a similar level of dispersion, while for weak interactions $\left(A_{P m}=70-75\right)$ the aggregation state depends on the potential acting between NPs.

In Fig. 18 the average number of NPs composing the aggregates is shown as function of monomer-NP Hamaker parameter for different mix parameters $f$. In this case, the cutoff adopted for the definition of NP-NP contacts is $r=6.25$. For a given Hamaker parameter, the average cluster size usually increases as $f$ decreases. There is, however, one notable exception in the case $A_{P m}=75$. The system with purely repulsive NP-NP interactions apparently produces the largest clusters. In these clusters, however, the particles are more loosely connected (smaller number of nearest neighbors), and quite likely they are also more mobile.

The potential acting between nanoparticles also affects the distribution of the number of nearest neighbors for each NP, as reported in Fig. 19. For the less favorable interactions between nanoparticles and polymer chains $\left[A_{P m}=75\right.$, in panel (a)] the shape of $B(n)$ remains roughly unchanged, but it shifts towards smaller values of $n$ when $f$ increases. For intermediate interactions $\left[A_{P m}=90\right.$, in panel (b)], the distribution sharply changes from $f=0.50$ and $f=1.00$. In the latter case, the nanoparticles are already isolated and well dispersed, in agreement with the evidence coming from the radial distribution functions and average cluster sizes. 
Potentials and morphology of polymer-nanoparticle blends

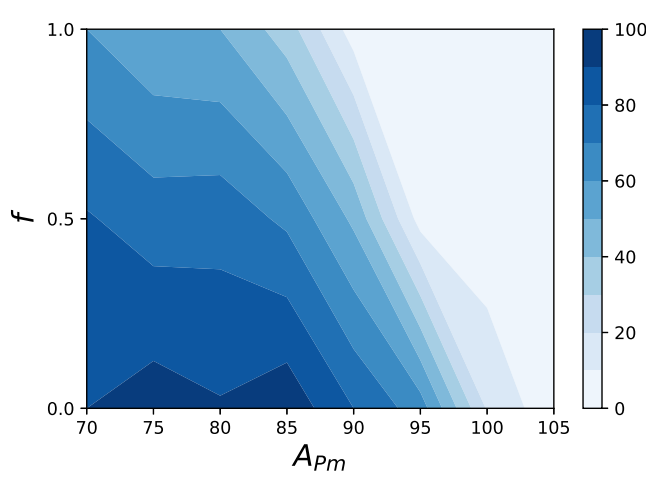

FIG. 17. Phase diagram for systems of type $\mathrm{L}$ with particle volume fraction $\phi \simeq 4.8 \%$, giving $g_{P P}^{1}$ as function of $A_{P m}$ and $f$.

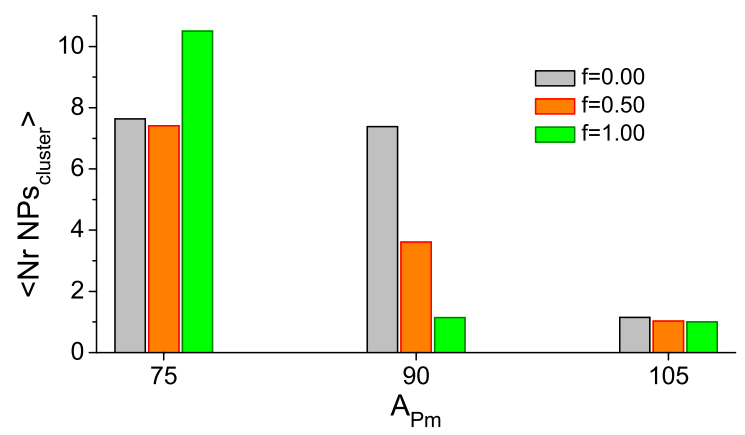

FIG. 18. Average number of NPs composing the aggregates for systems of type L.

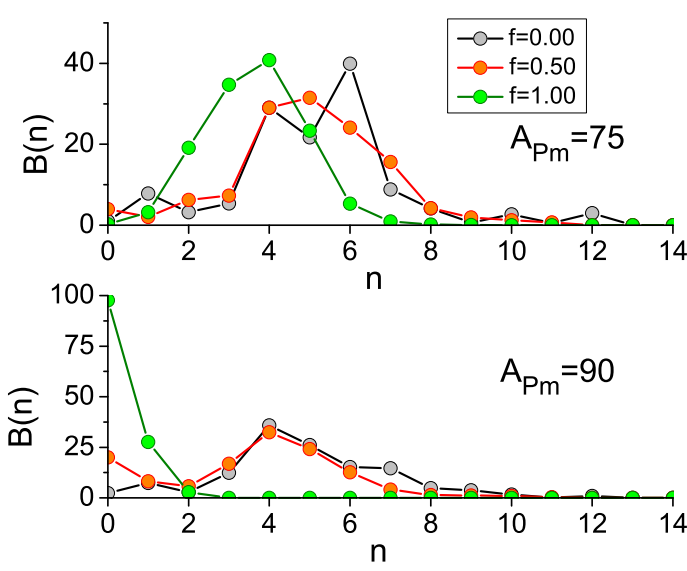

FIG. 19. Average distributions of nearest neighbors per NP as obtained for different NP-NP interactions for system L. Panel (a): system with a weak monomer-NP interaction $\left(A_{P m}=75\right)$. Panel (b): system with an intermediate monomer-NP interaction $\left(A_{P m}=90\right)$. 


\section{CLOSING REMARKS}

The morphology of polymer nanocomposites is a key feature for their performance. Achieving a good degree of dispersion may be not easy, but it is usually beneficial in comparison with poorly dispersed states. Intermediate situations, such as those characterized nanoparticle clusters of reasonably controlled size and shape (e.g., linear or branched aggregates), may be even superior for some applications. Molecular simulations, especially at the coarse-grained level, can help us understand the formation, stability and properties of such nanocomposite morphologies. Ideally, coarse-grained models should flexible enough to capture the salient features of real systems, but simple enough to be amenable to theoretical analyses and to allow a systematic exploration of their parameter space. In this work, we have introduced one such "simple but flexible" model of polymer nanocomposites. The flexibility comes from the "mixing parameter" $f$, which can be used to tune the interparticle interactions between the purely repulsive and fully attractive limits ( $f=1.0$ and $f=0.0$, respectively). We have studied the effect of this parameter, in combination with the Hamaker constant for polymer-nanoparticle interactions, on the equilibrium morphologies of blends with different particle sizes. We find that it is possible to obtain both well-dispersed and aggregated morphologies—even in presence of strong interparticle attractions—by changing the strength of the polymer-nanoparticle interactions. However, the $f$ parameter allows some extra fine tuning of these morphologies. Both the final aggregated morphologies, and the sharpness of the transition to the dispersed state, are quite sensitive to it. In the future we plan to explore further these systems, characterizing the dynamical as well as the equilibrium properties of the nanocomposites and looking at their behavior at interfaces, as in free standing nanocomposite films or following deposition on solid substrates. ${ }^{24}$ Finally, we point out that our strategy for mixing repulsive and attractive potentials is general and may be applied to also to other situations. This includes the simple Lennard-Jones potential, adding one degree of freedom $(f)$ to its menu of adjustable parameters (see ref.44 for recent related work).

\section{ACKNOWLEDGMENTS}

The authors acknowledge the PRIN2015 program for financial support (project no. 2015XJA9NT), and CINECA for high performance computing resources through the ISCRA initiative. GR thanks Dr. Amalie Frishknecht for useful discussions, in the early stages of this research. 
Supplementary material: Python code to generate the tabulated potentials and forces, in LAMMPS-compatible format.

Data availability statement: The data that support the findings of this study are available from the corresponding author upon reasonable request.

\section{REFERENCES}

${ }^{1}$ A. C. Balazs, T. Emrick, and T. P. Russell, "Nanoparticle polymer composites: Where two small worlds meet," Science 314, 1107-1110 (2006).

${ }^{2}$ S. K. Kumar, B. C. Benicewicz, R. A. Vaia, and K. I. Winey, "50th anniversary perspective: Are polymer nanocomposites practical for applications?” Macromolecules 50, 714-731 (2017).

${ }^{3}$ J. Jancar, J. Douglas, F. W. Starr, S. Kumar, P. Cassagnau, A. Lesser, S. S. Sternstein, and M. Buehler, "Current issues in research on structure-property relationships in polymer nanocomposites," Polymer 51, 3321-3343 (2010).

${ }^{4}$ C.-C. Lin, E. Parrish, and R. J. Composto, "Macromolecule and particle dynamics in confined media," Macromolecules 49, 5755-5772 (2016).

${ }^{5} \mathrm{G}$. Raos, "Application of the christensen-lo model to the reinforcement of elastomers by fractal fillers," Macromolecular theory and simulations 12, 17-23 (2003).

${ }^{6}$ D. F. Moyano and V. M. Rotello, "Nano meets biology: structure and function at the nanoparticle interface," Langmuir 27, 10376-10385 (2011).

${ }^{7}$ C. K. Payne, "A protein corona primer for physical chemists," The Journal of chemical physics 151, 130901 (2019).

${ }^{8}$ Y. Ju-Nam and J. R. Lead, "Manufactured nanoparticles: An overview of their chemistry, interactions and potential environmental implications," Science of the Total Environment 400 , 396-414 (2008).

${ }^{9}$ R. A. Vaia and J. F. Maguire, "Polymer nanocomposites with prescribed morphology: Going beyond nanoparticle-filled polymers," Chemistry of Materials 19, 2736-2751 (2007).

${ }^{10}$ S. K. Kumar, N. Jouault, B. Benicewicz, and T. Neely, "Nanocomposites with polymer grafted nanoparticles," Macromolecules 46, 3199-3214 (2013).

${ }^{11}$ J. Israelachvili, Intermolecular and Surface Forces (Academic Press, 1992).

${ }^{12}$ G. Allegra, G. Raos, and M. Vacatello, "Theories and simulations of polymer-based nanocomposites: From chain statistics to reinforcement," Progress in Polymer Science 33, 683-731 
(2008).

${ }^{13}$ V. Ganesan and A. Jayaraman, "Theory and simulation studies of effective interactions, phase behavior and morphology in polymer nanocomposites," Soft Matter 10, 13-38 (2014).

${ }^{14}$ Y. N. Pandey, G. J. Papakonstantopoulos, and M. Doxastakis, "Polymer/Nanoparticle Interactions: Bridging the Gap,” Macromolecules 46, 5097-5106 (2013).

${ }^{15}$ H. Heinz, T. J. Lin, R. Kishore Mishra, and F. S. Emami, “Thermodynamically consistent force fields for the assembly of inorganic, organic, and biological nanostructures: The INTERFACE force field," Langmuir 29, 1754-1765 (2013), arXiv:1408.1149.

${ }^{16}$ S. J. Plimpton and A. P. Thompson, "Computational aspects of many-body potentials," MRS Bulletin 37, 513-521 (2012).

${ }^{17}$ D. Meng, S. K. Kumar, J. M. D. Lane, and G. S. Grest, "Effective interactions between grafted nanoparticles in a polymer matrix," Soft Matter 8, 5002-5010 (2012).

${ }^{18}$ G. Munaò, A. Correa, A. Pizzirusso, and G. Milano, "On the calculation of the potential of mean force between atomistic nanoparticles," The European Physical Journal E 41, 38 (2018).

${ }^{19}$ G. Munaò, A. Pizzirusso, A. Kalogirou, A. De Nicola, T. Kawakatsu, F. Müller-Plathe, and G. Milano, "Molecular structure and multi-body potential of mean force in silica-polystyrene nanocomposites," Nanoscale 10, 21656-21670 (2018).

${ }^{20}$ C. Liepold, A. Smith, B. Lin, J. de Pablo, and S. A. Rice, "Pair and many-body interactions between ligated au nanoparticles,” The Journal of chemical physics 150, 044904 (2019).

${ }^{21}$ J. B. Hooper and K. S. Schweizer, "Contact aggregation, bridging, and steric stabilization in dense polymer-particle mixtures," Macromolecules 38, 8858-8869 (2005).

${ }^{22}$ L. M. Hall and K. S. Schweizer, "Many body effects on the phase separation and structure of dense polymer-particle melts." The Journal of chemical physics 128, 234901 (2008).

${ }^{23}$ A. L. Frischknecht and A. Yethiraj, "Two- and three-body interactions among nanoparticles in a polymer melt.” The Journal of chemical physics 134, 174901 (2011).

${ }^{24}$ A. L. Frischknecht, V. Padmanabhan, and M. E. Mackay, "Surface-induced phase behavior of polymer/nanoparticle blends with attractions.” The Journal of chemical physics 136, 164904 (2012).

${ }^{25}$ S. Egorov, "Anomalous nanoparticle diffusion in polymer solutions and melts: A mode-coupling theory study," The Journal of chemical physics 134, 084903 (2011).

${ }^{26} \mathrm{U}$. Yamamoto and K. S. Schweizer, "Theory of nanoparticle diffusion in unentangled and entangled polymer melts," The Journal of Chemical Physics 135, 224902 (2011). 
${ }^{27}$ K. Kremer and G. S. Grest, "Dynamics of entangled linear polymer melts: A moleculardynamics simulation,” The Journal of Chemical Physics 92, 5057-5086 (1990).

${ }^{28}$ J.-P. Hansen and I. R. McDonald, Theory of simple liquids (Elsevier, 1990).

${ }^{29}$ M. P. Allen and D. J. Tildesley, Computer simulation of liquids, 2nd edition (Oxford University Press, 2017).

${ }^{30}$ A. Patti, "Molecular dynamics of spherical nanoparticles in dense polymer melts," Journal of Physical Chemistry B 118, 3731-3742 (2014).

${ }^{31}$ Y. Li, M. Kröger, and W. K. Liu, "Dynamic structure of unentangled polymer chains in the vicinity of non-attractive nanoparticles," Soft Matter 10, 1723 (2014).

${ }^{32}$ K. Hagita, H. Morita, M. Doi, and H. Takano, "Coarse-Grained Molecular Dynamics Simulation of Filled Polymer Nanocomposites under Uniaxial Elongation,” Macromolecules 49, 1972-1983 (2016).

${ }^{33}$ D. Gersappe, "Molecular Mechanisms of Failure in Polymer Nanocomposites," Physical Review Letters 89, 058301 (2002).

${ }^{34}$ J. Liu, D. Cao, L. Zhang, and W. Wang, "Time-Temperature and Time-Concentration Superposition of Nanofilled Elastomers: A Molecular Dynamics Study,” , 2831-2842 (2009).

${ }^{35}$ V. Sorichetti, V. Hugouvieux, and W. Kob, "Structure and Dynamics of a Polymer-Nanoparticle Composite: Effect of Nanoparticle Size and Volume Fraction," Macromolecules 51, 5375-5391 (2018).

${ }^{36}$ N. Molinari, A. Sutton, and A. Mostofi, "Mechanisms of reinforcement in polymer nanocomposites," Physical Chemistry Chemical Physics 20, 23085-23094 (2018).

${ }^{37}$ H. C. Hamaker, "The london—van der waals attraction between spherical particles," physica $\mathbf{4}$, 1058-1072 (1937).

${ }^{38}$ R. Everaers and M. Ejtehadi, "Interaction potentials for soft and hard ellipsoids," Physical Review E 67, 041710 (2003).

${ }^{39}$ D. Meng, S. K. Kumar, S. Cheng, and G. S. Grest, "Simulating the miscibility of nanoparticles and polymer melts," Soft Matter 9, 5417-5427 (2013).

${ }^{40}$ D. Meng, S. K. Kumar, J. M. D. Lane, and G. S. Grest, "Effective interactions between grafted nanoparticles in a polymer matrix," Soft Matter 8, 5002-5010 (2012).

${ }^{41}$ J. D. Weeks, D. Chandler, and H. C. Andersen, "Role of repulsive forces in determining the equilibrium structure of simple liquids," The Journal of Chemical Physics 54, 5237-5247 (1971).

${ }^{42}$ D. Chandler, J. D. Weeks, and H. C. Andersen, "Van der waals picture of liquids, solids, and 
Potentials and morphology of polymer-nanoparticle blends

phase transformations," Science 220, 787-794 (1983).

${ }^{43}$ S. Plimpton, "Fast parallel algorithms for short-range molecular dynamics," Journal of computational physics 117, 1-19 (1995).

${ }^{44}$ X. Wang, S. Ramírez-Hinestrosa, J. Dobnikar, and D. Frenkel, "The Lennard-Jones potential: when (not) to use it," Physical Chemistry Chemical Physics (2020), 10.1039/C9CP05445F, arXiv:1910.05746.

${ }^{45}$ M. Kröger, “Simple models for complex nonequilibrium fluids," Physics reports 390, 453-551 (2004).

${ }^{46}$ V. A. Froltsov, M. Klüppel, and G. Raos, "Molecular dynamics simulation of rupture in glassy polymer bridges within filler aggregates," Physical Review E 86, 041801 (2012).

${ }^{47}$ D. Frenkel, "Order through entropy," Nature materials 14, 9 (2014).

${ }^{48}$ A. Karatrantos, R. J. Composto, K. I. Winey, M. Kröger, and N. Clarke, "Modeling of Entangled Polymer Diffusion in Melts and Nanocomposites: A Review," Polymers 11, 876 (2019).

${ }^{49}$ G. Raos and J. Idé, "Impact of Interaction Strength and Surface Heterogeneity on the Dynamics of Adsorbed Polymers," ACS Macro Letters 3, 721-726 (2014).

${ }^{50}$ R. Pastore and G. Raos, "Glassy dynamics of a polymer monolayer on a heterogeneous disordered substrate," Soft Matter 11, 8083-8091 (2015). 


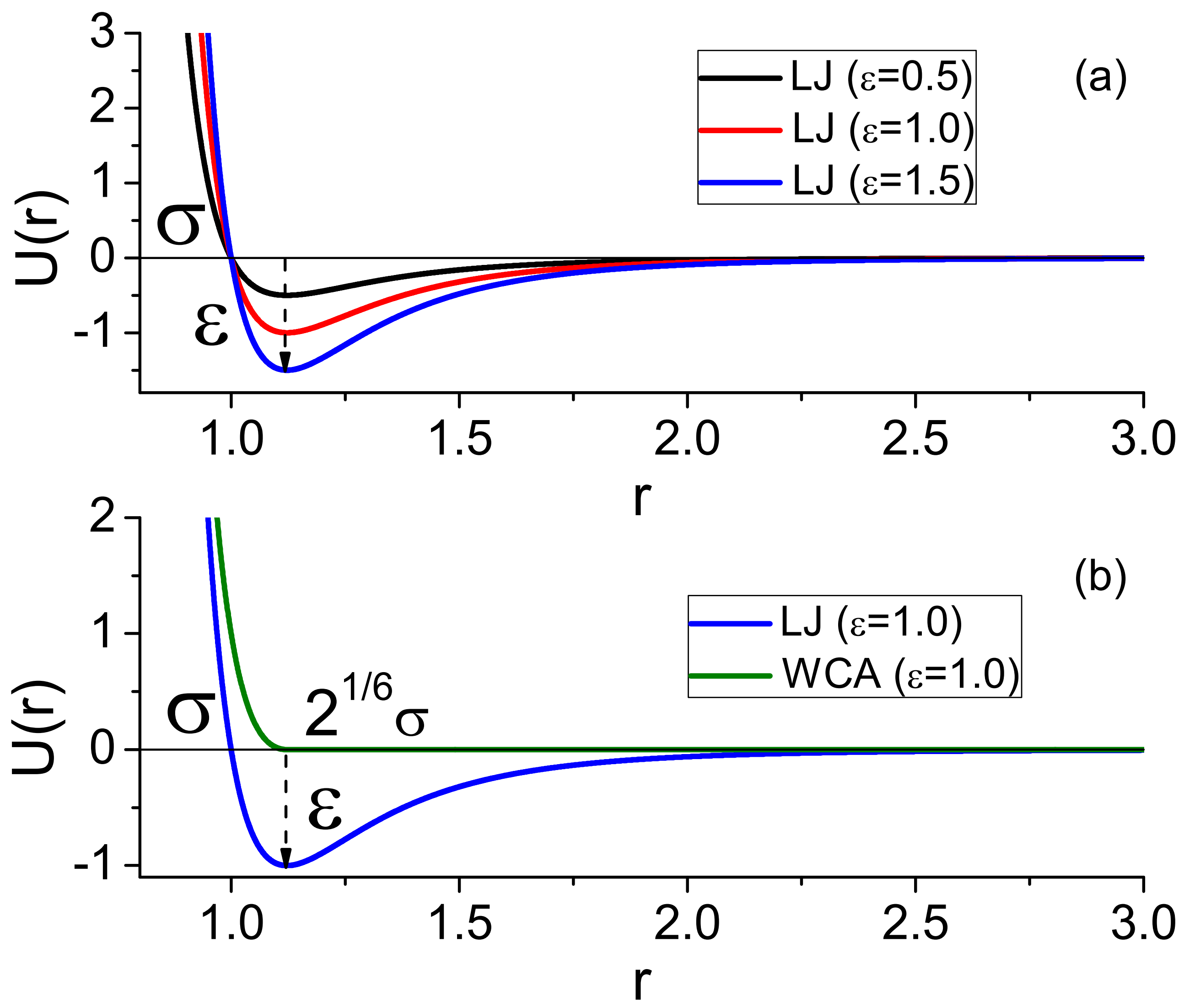




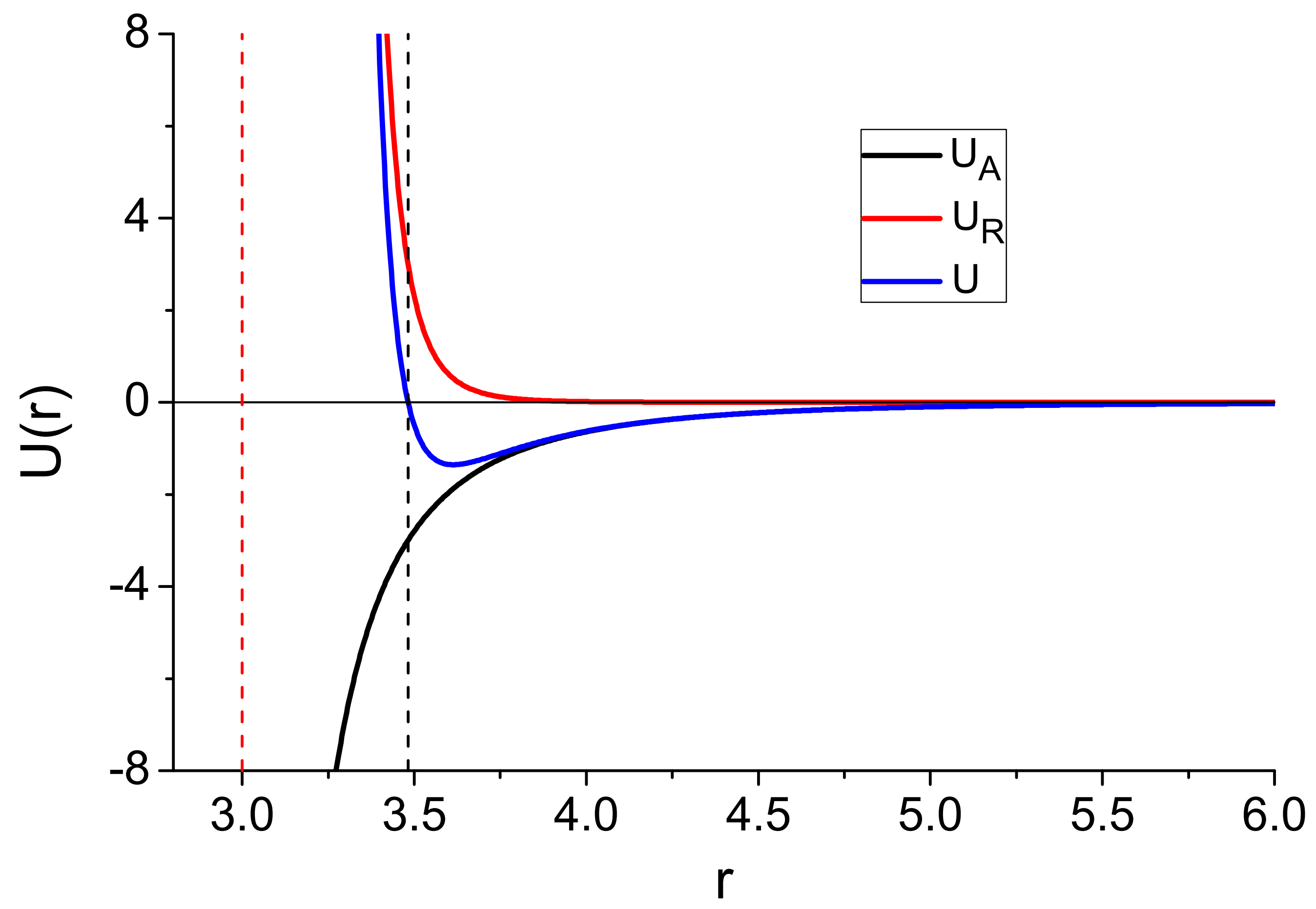




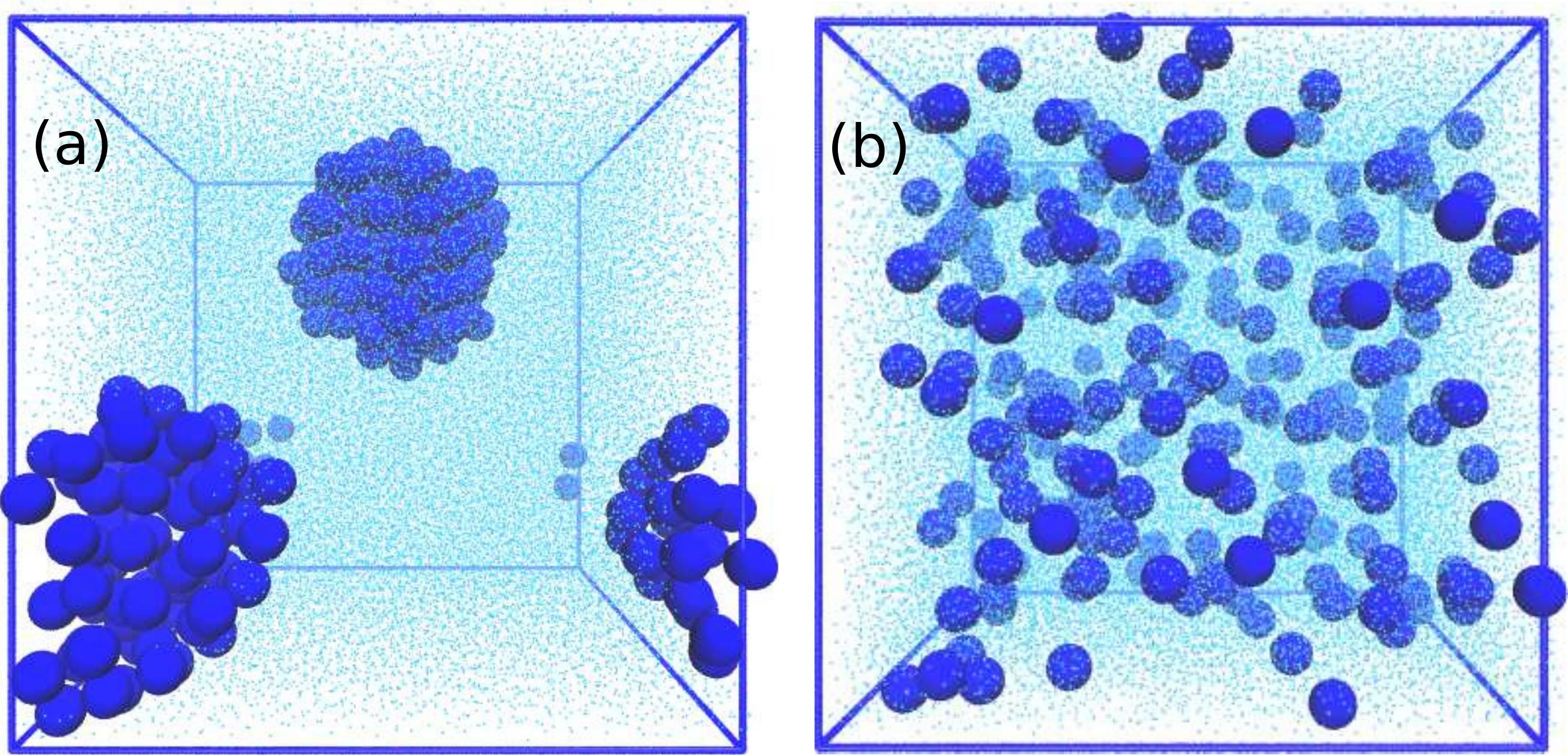




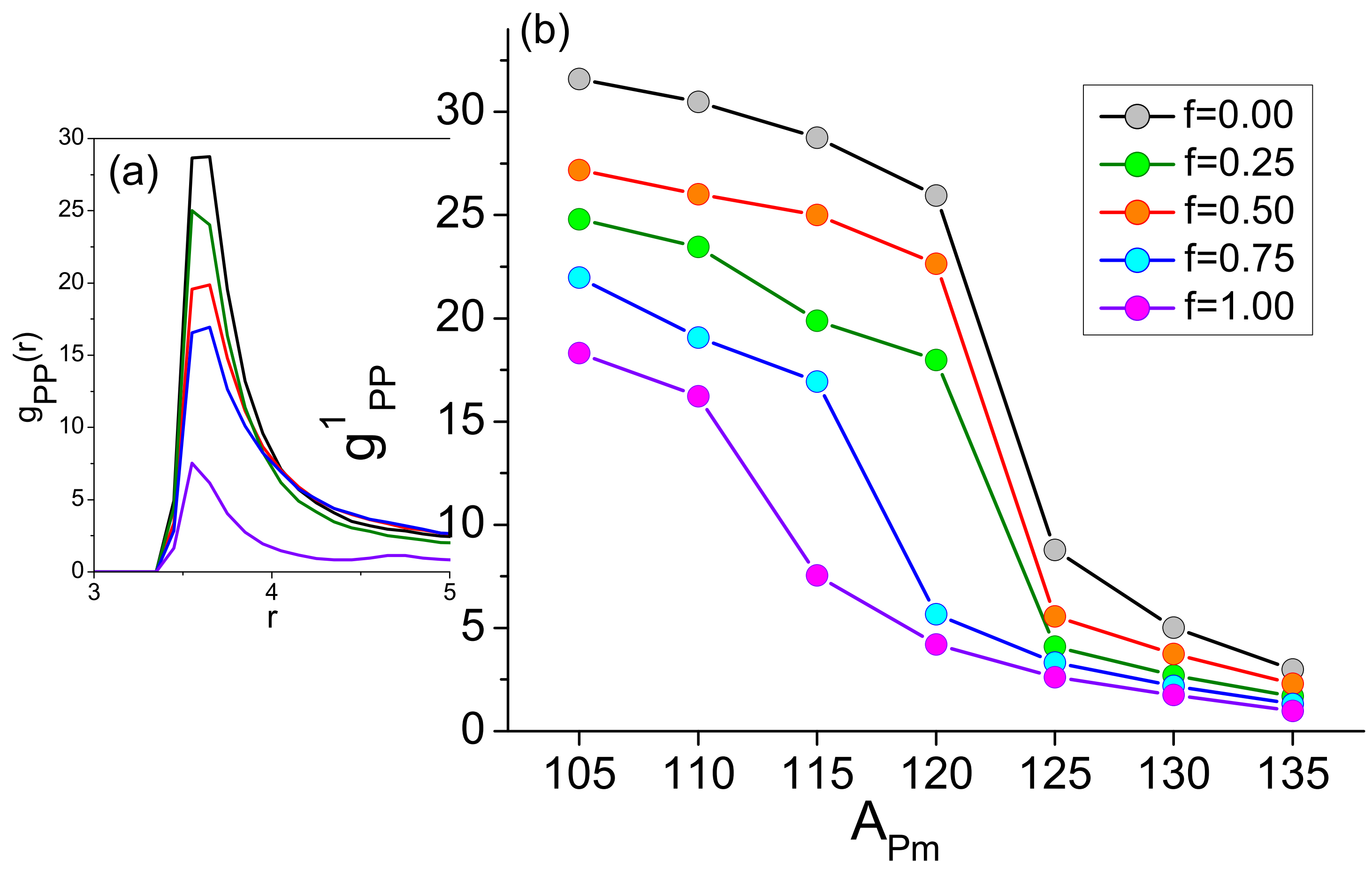




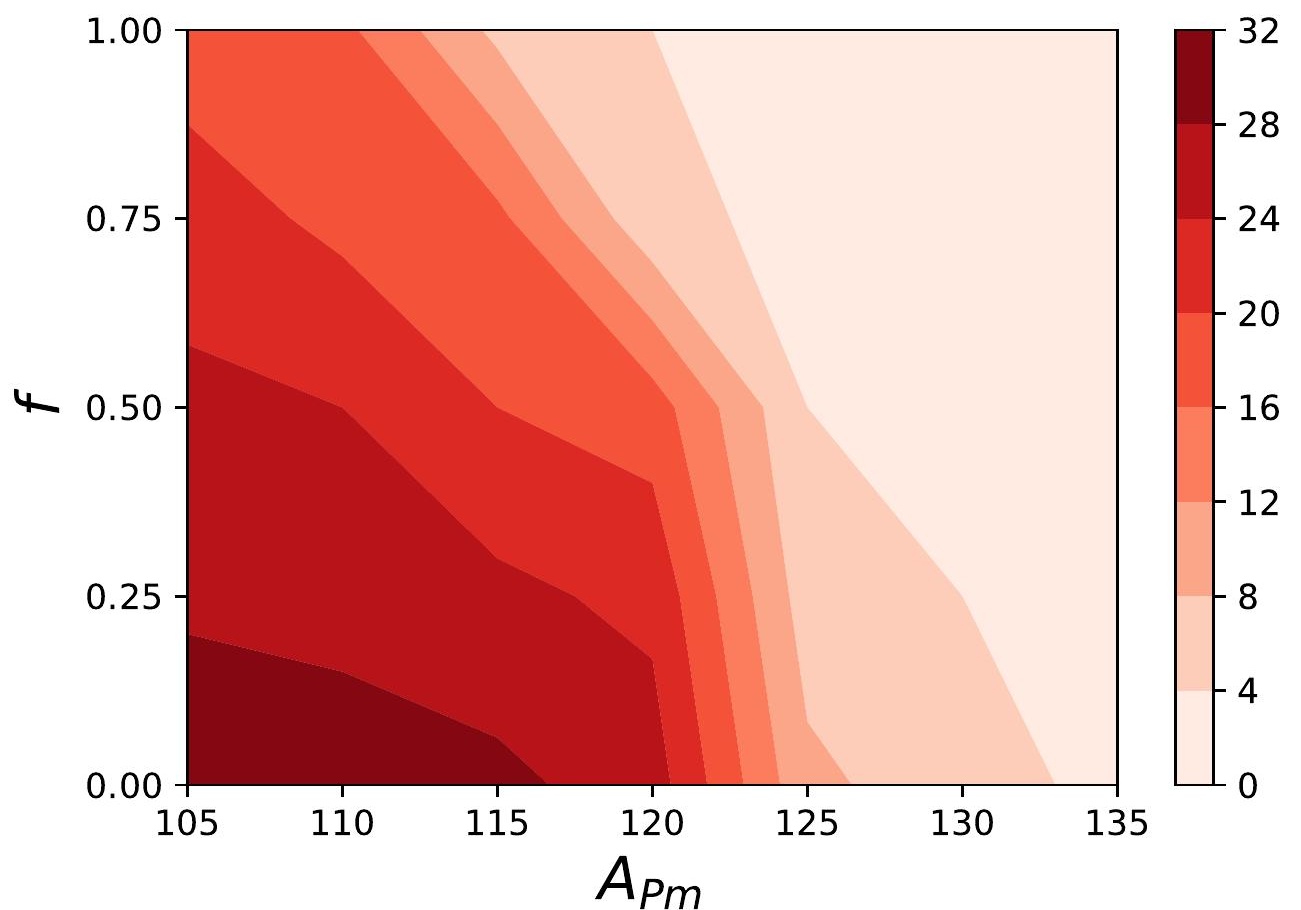



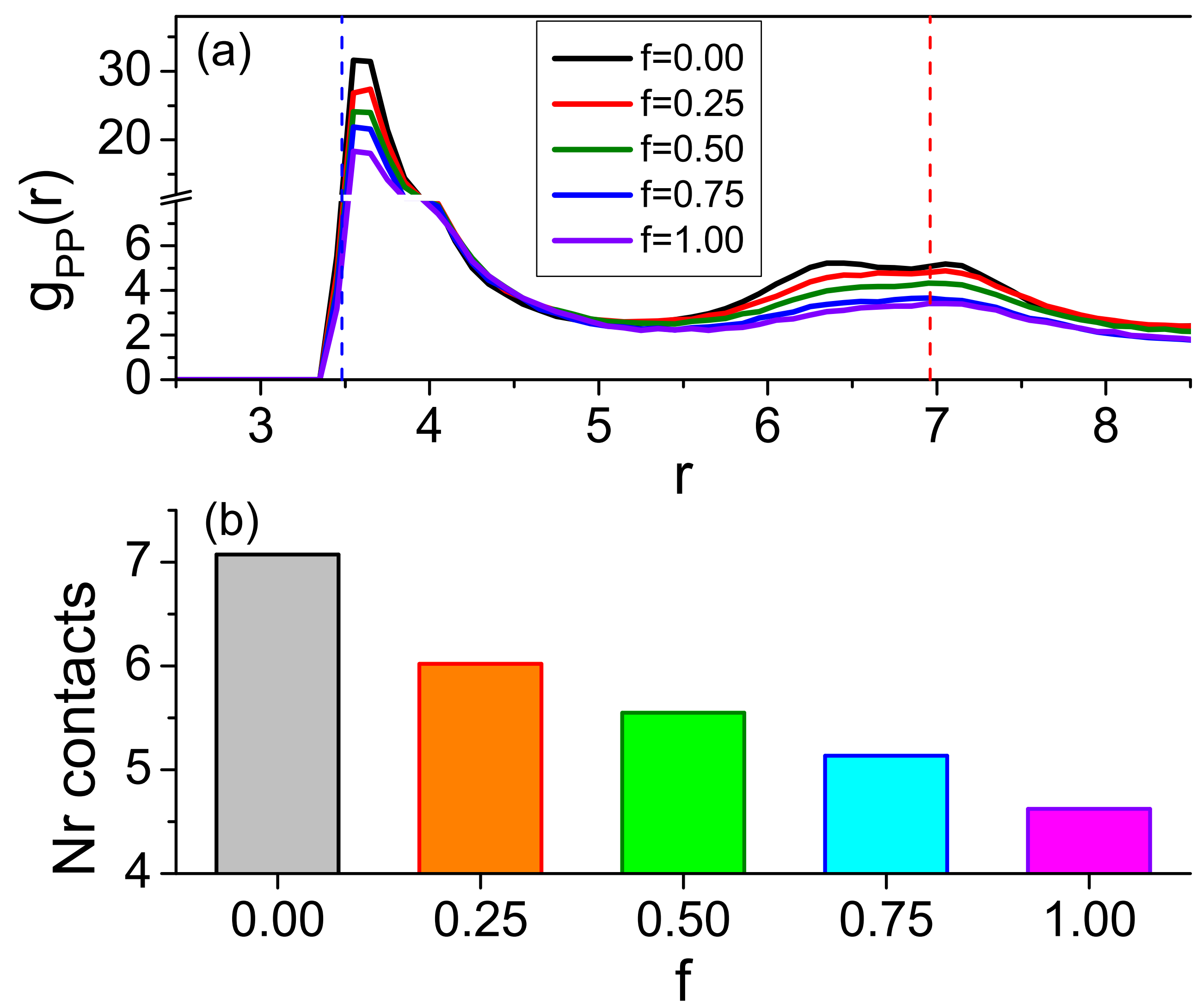


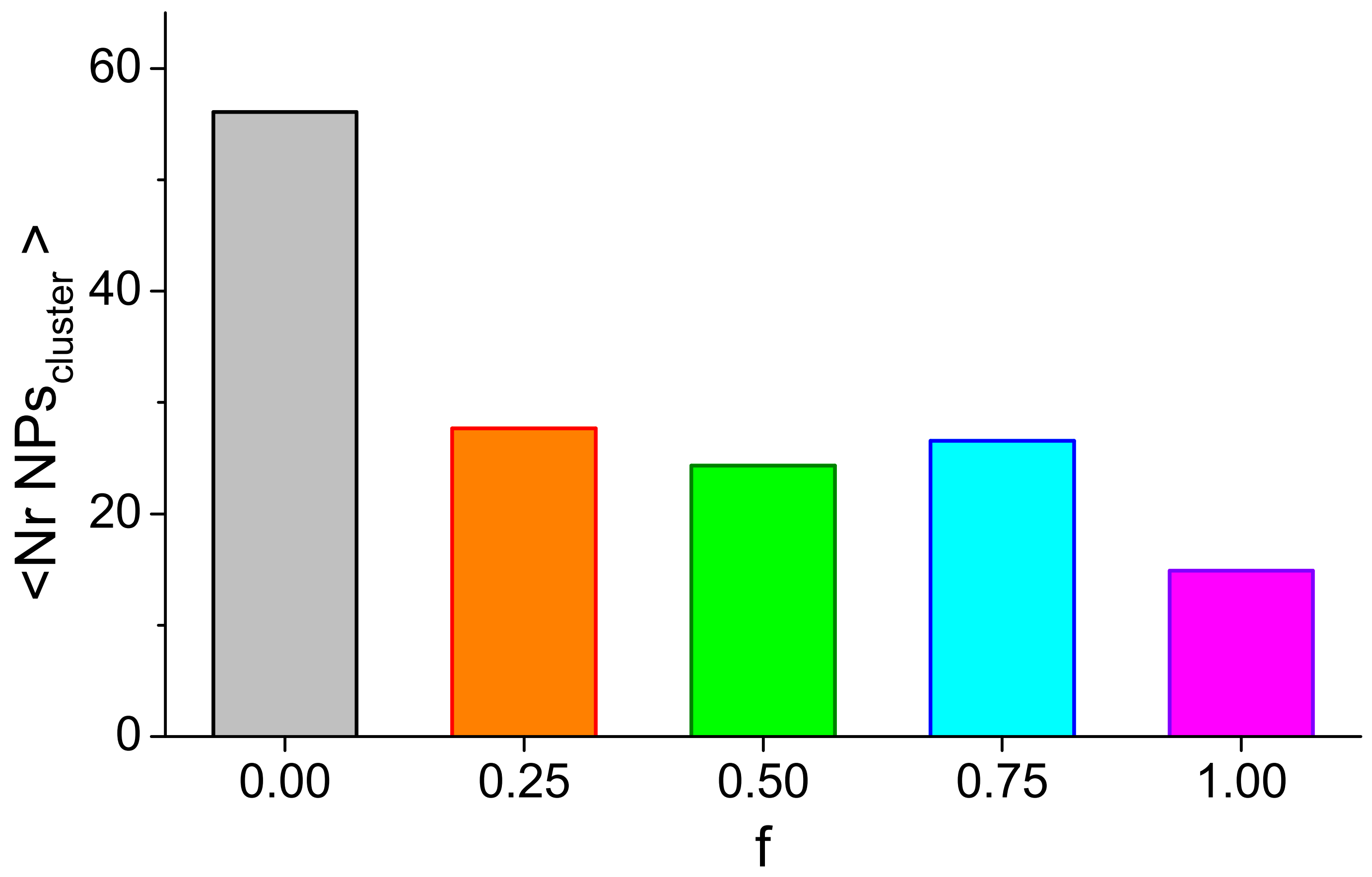




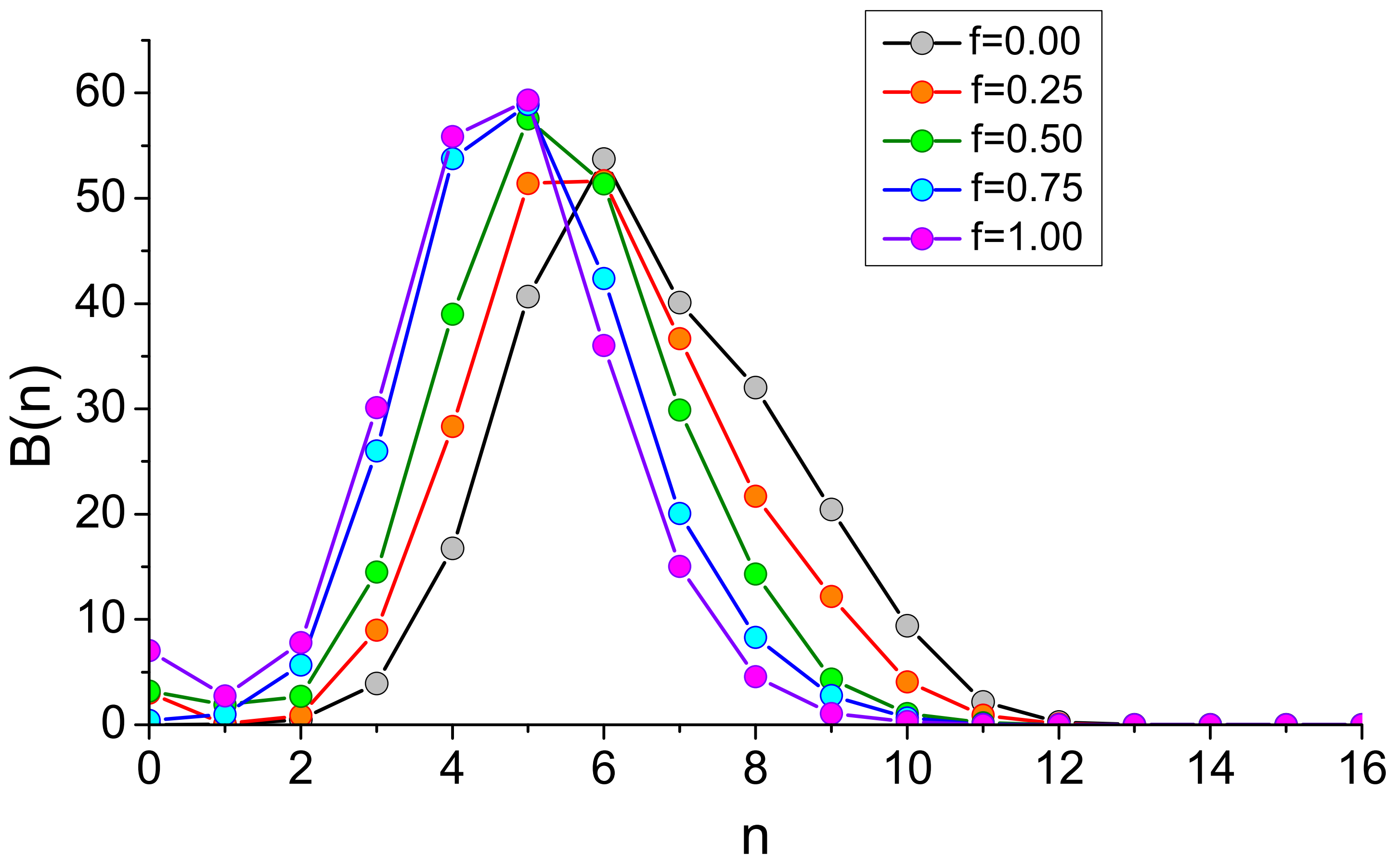




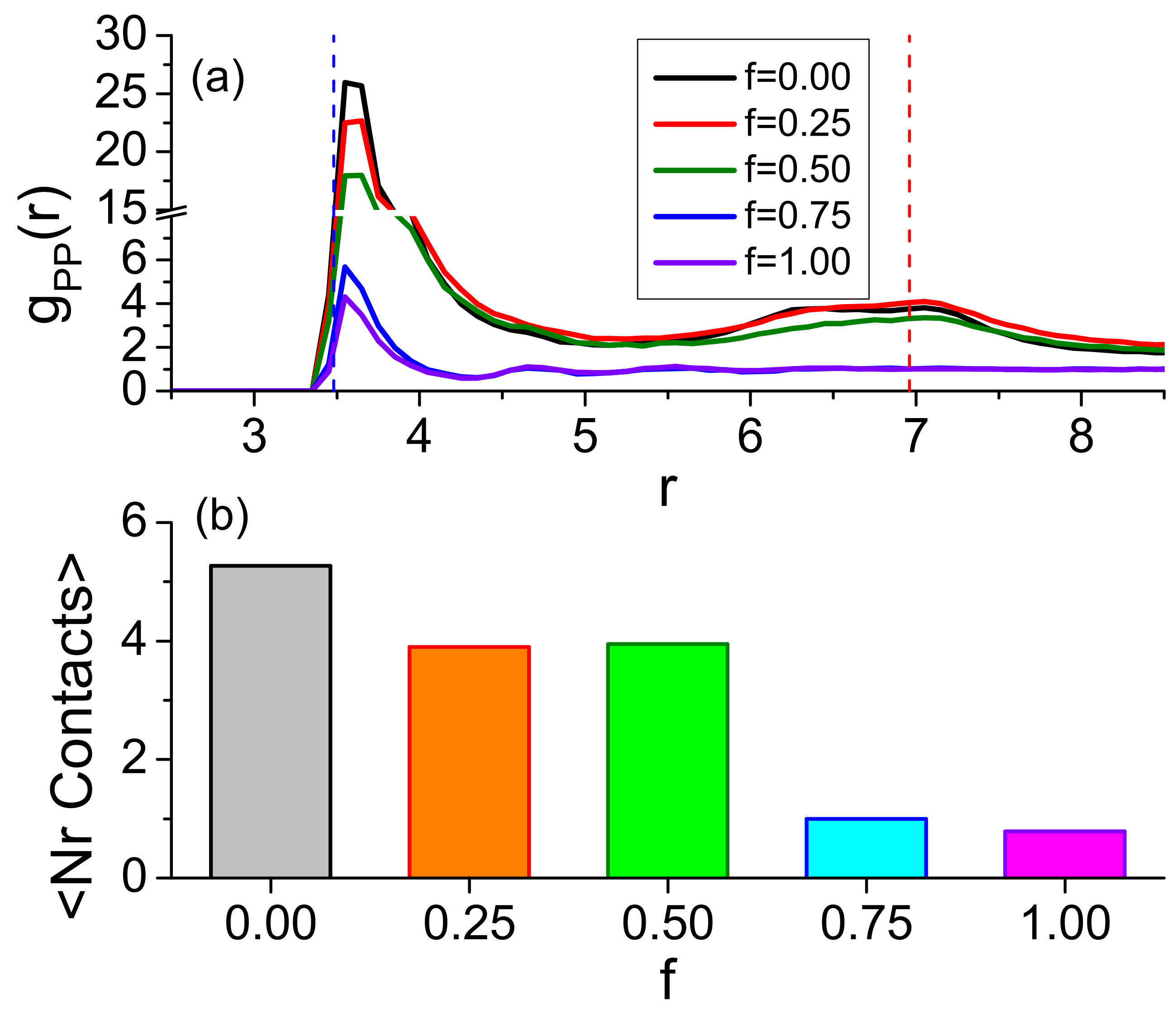



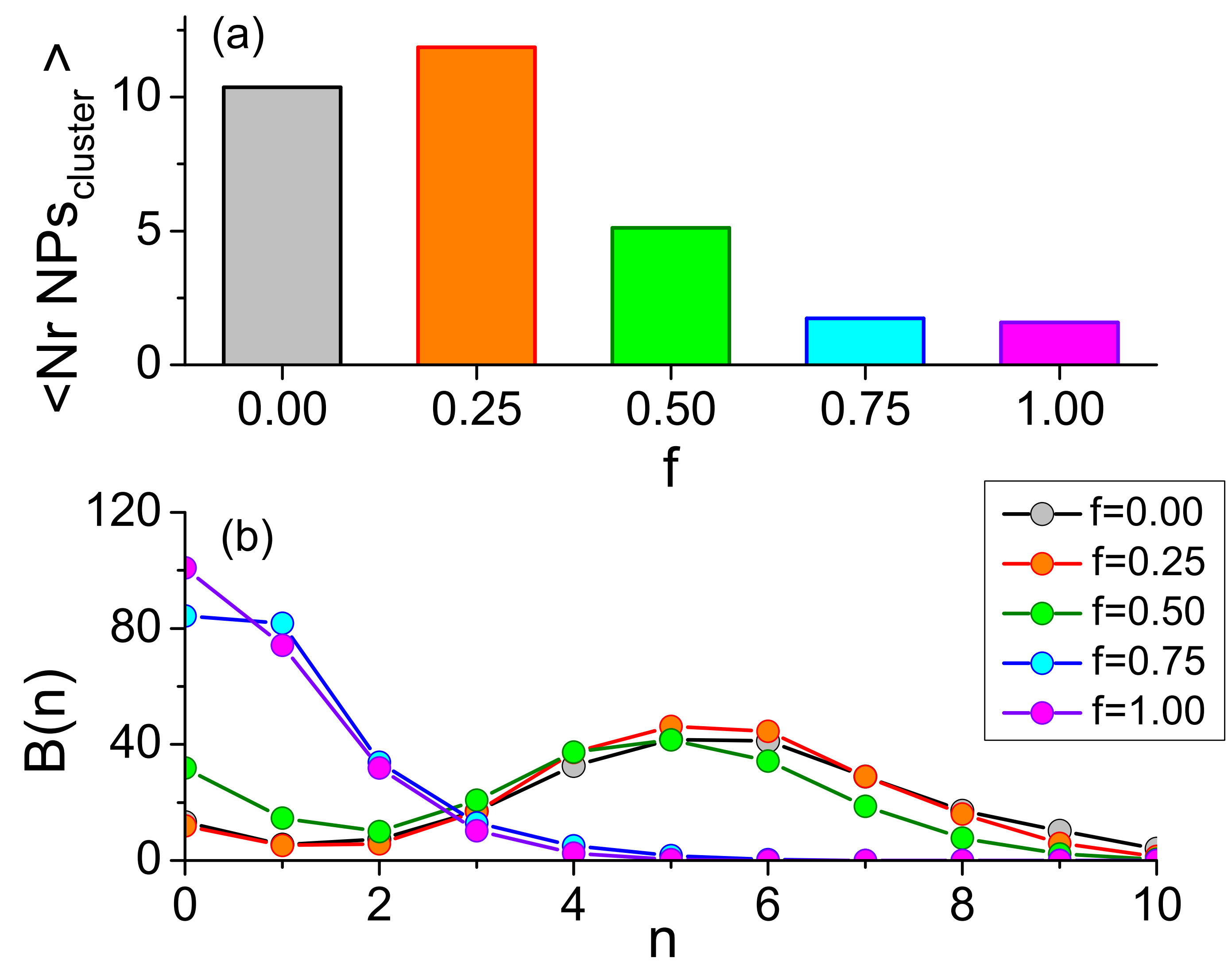


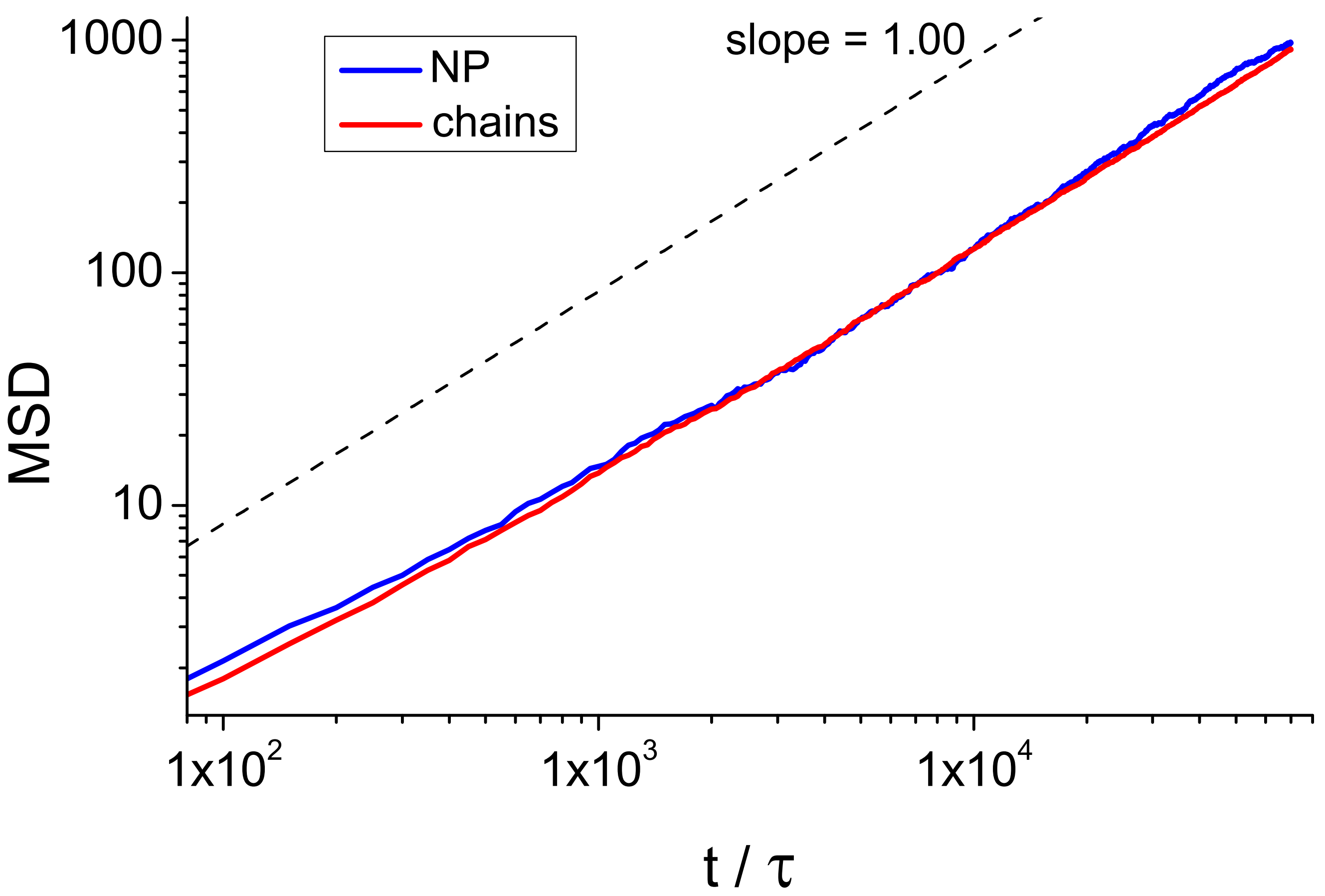



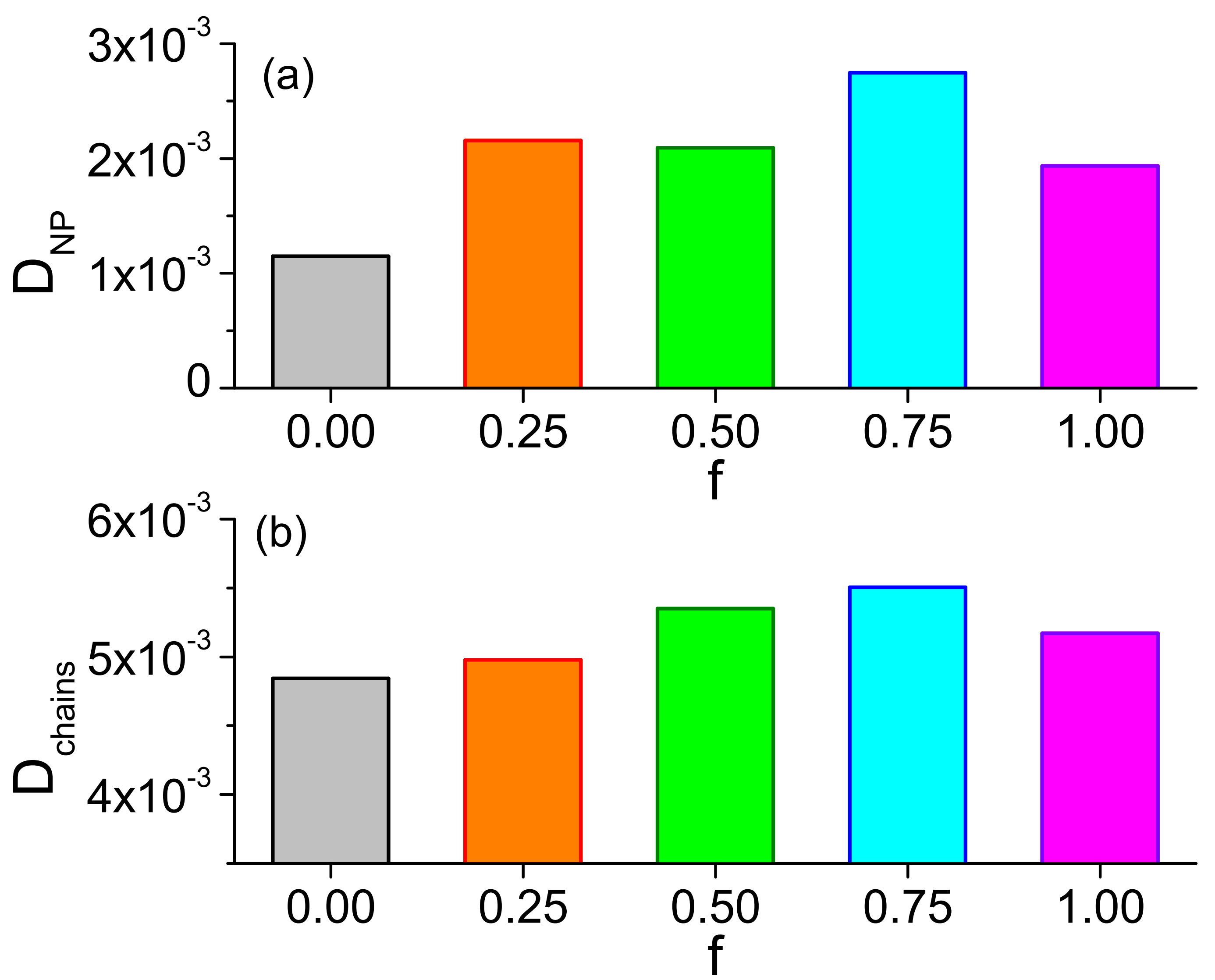

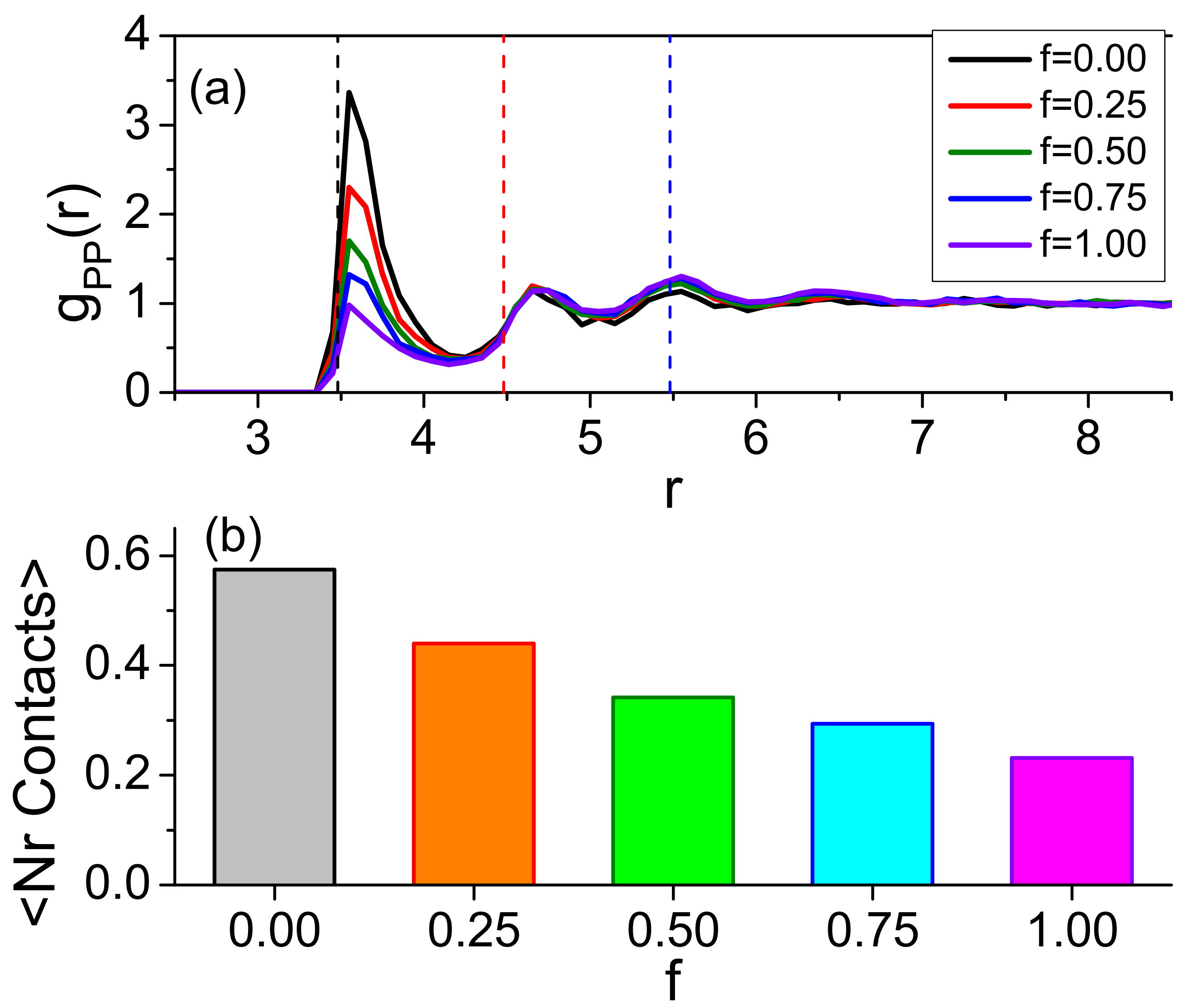

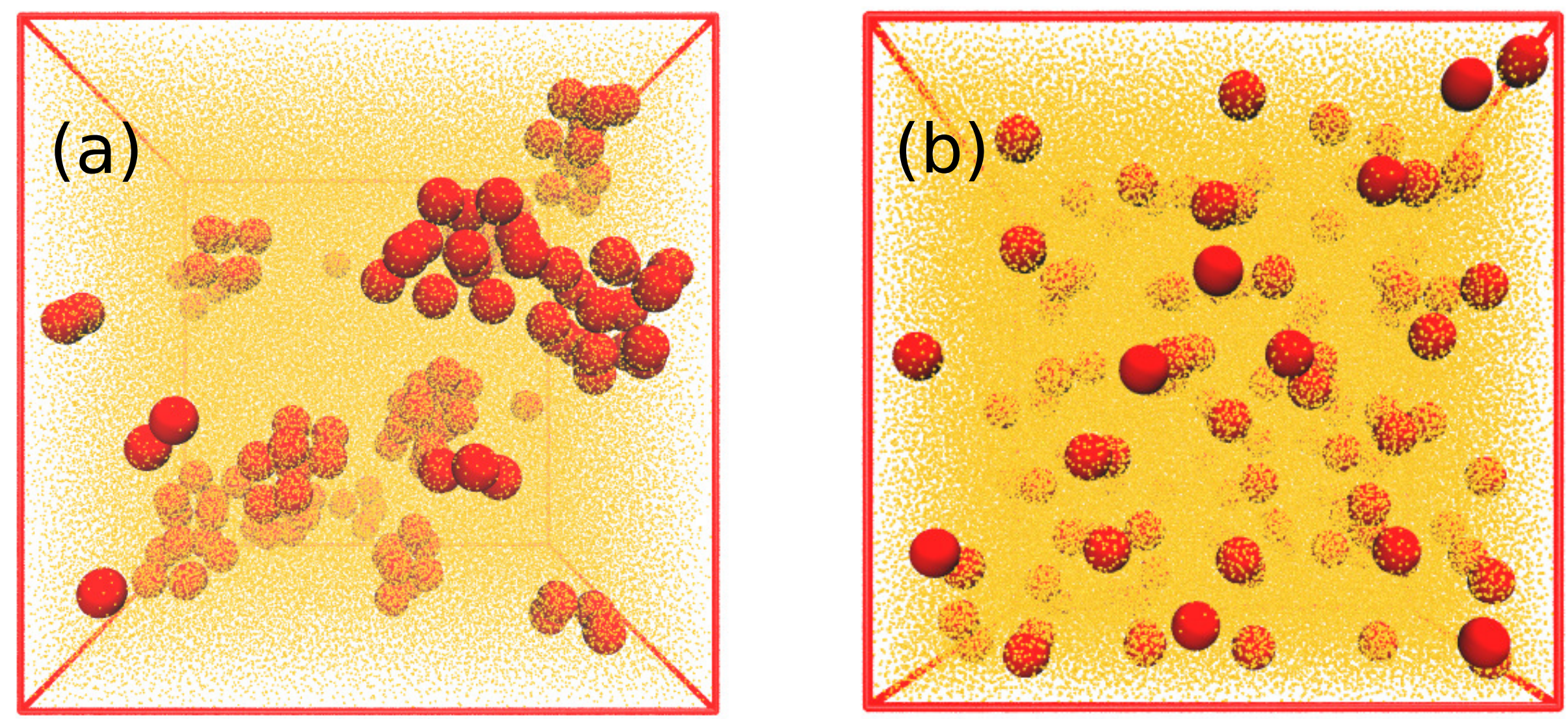


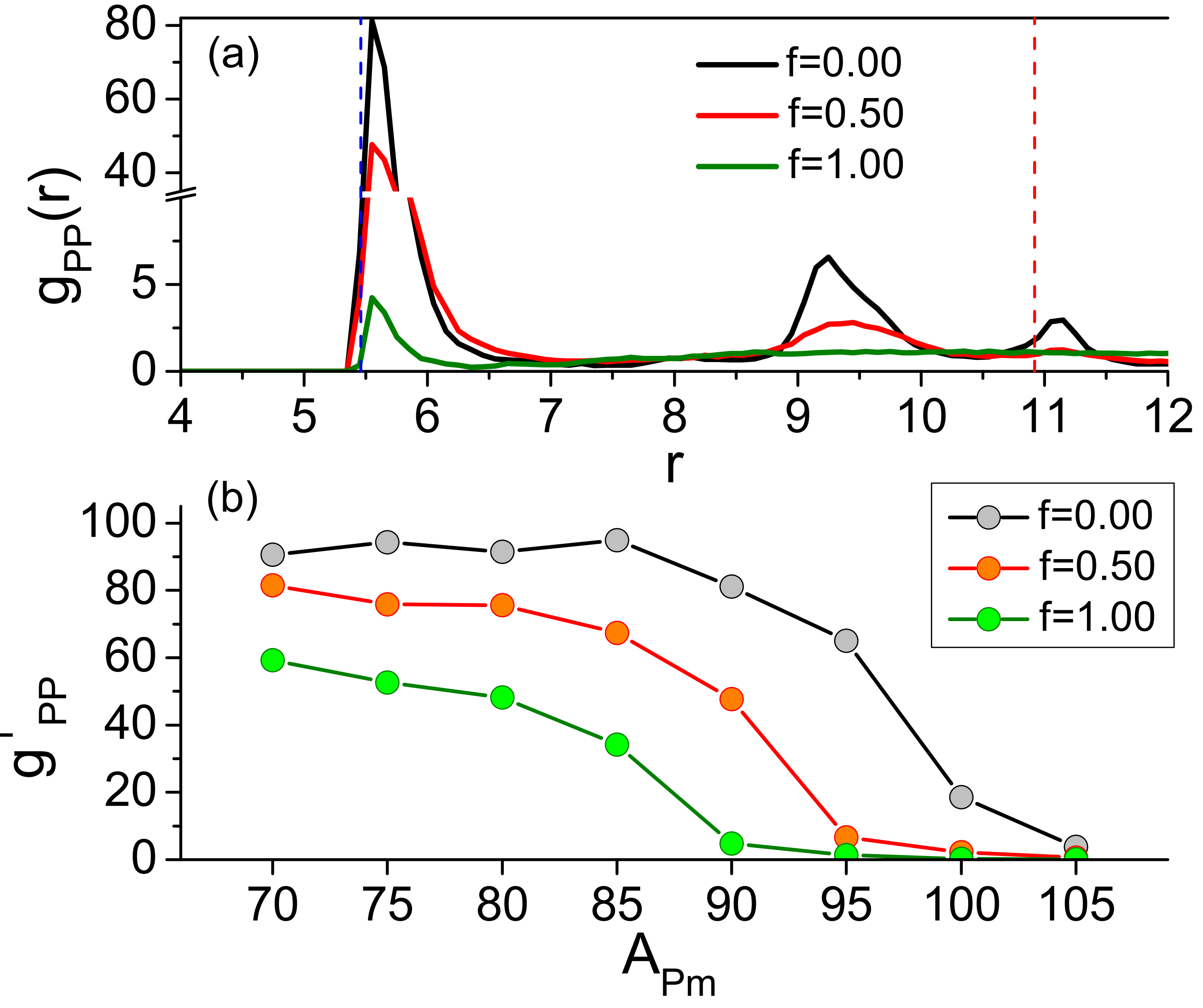




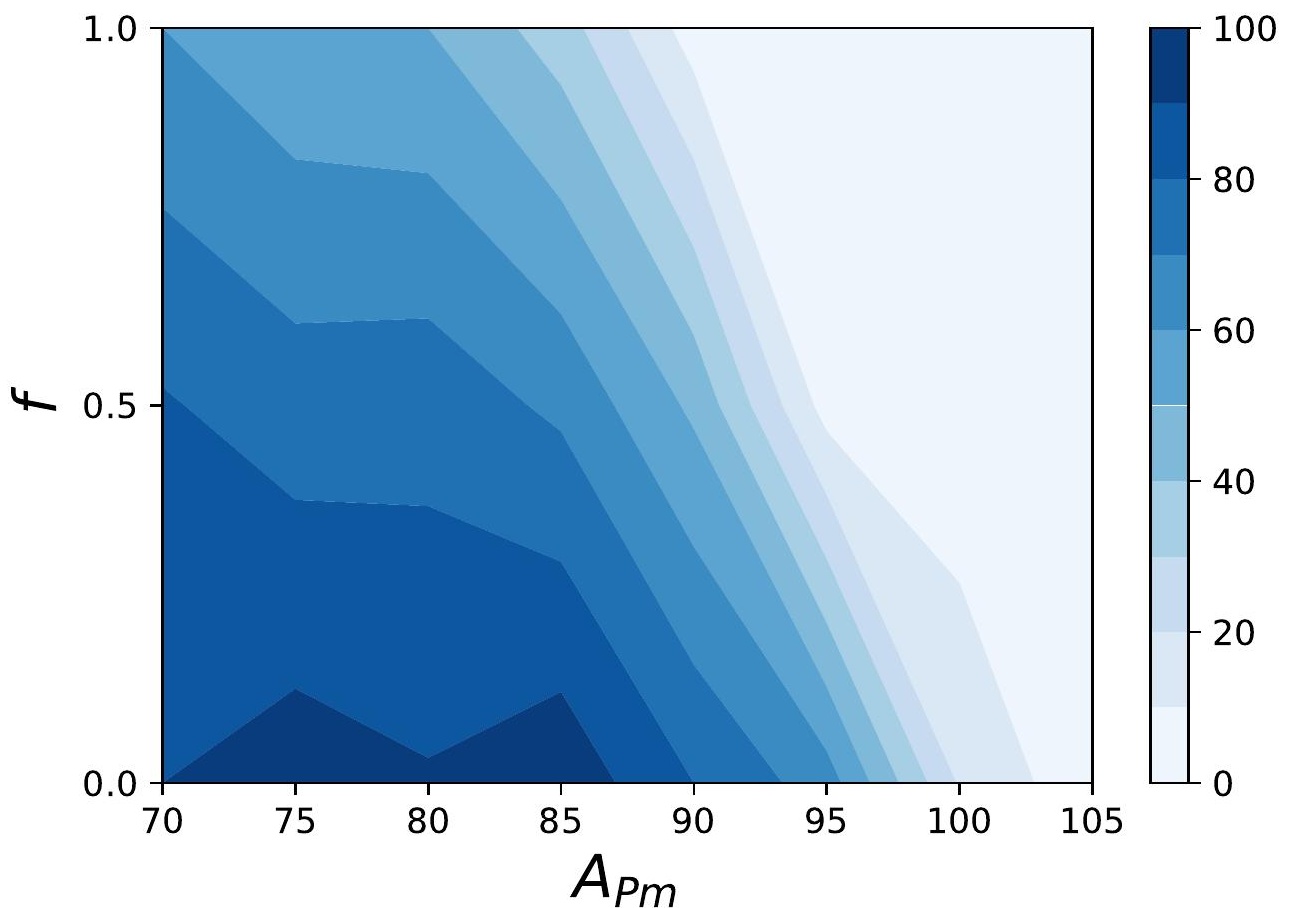




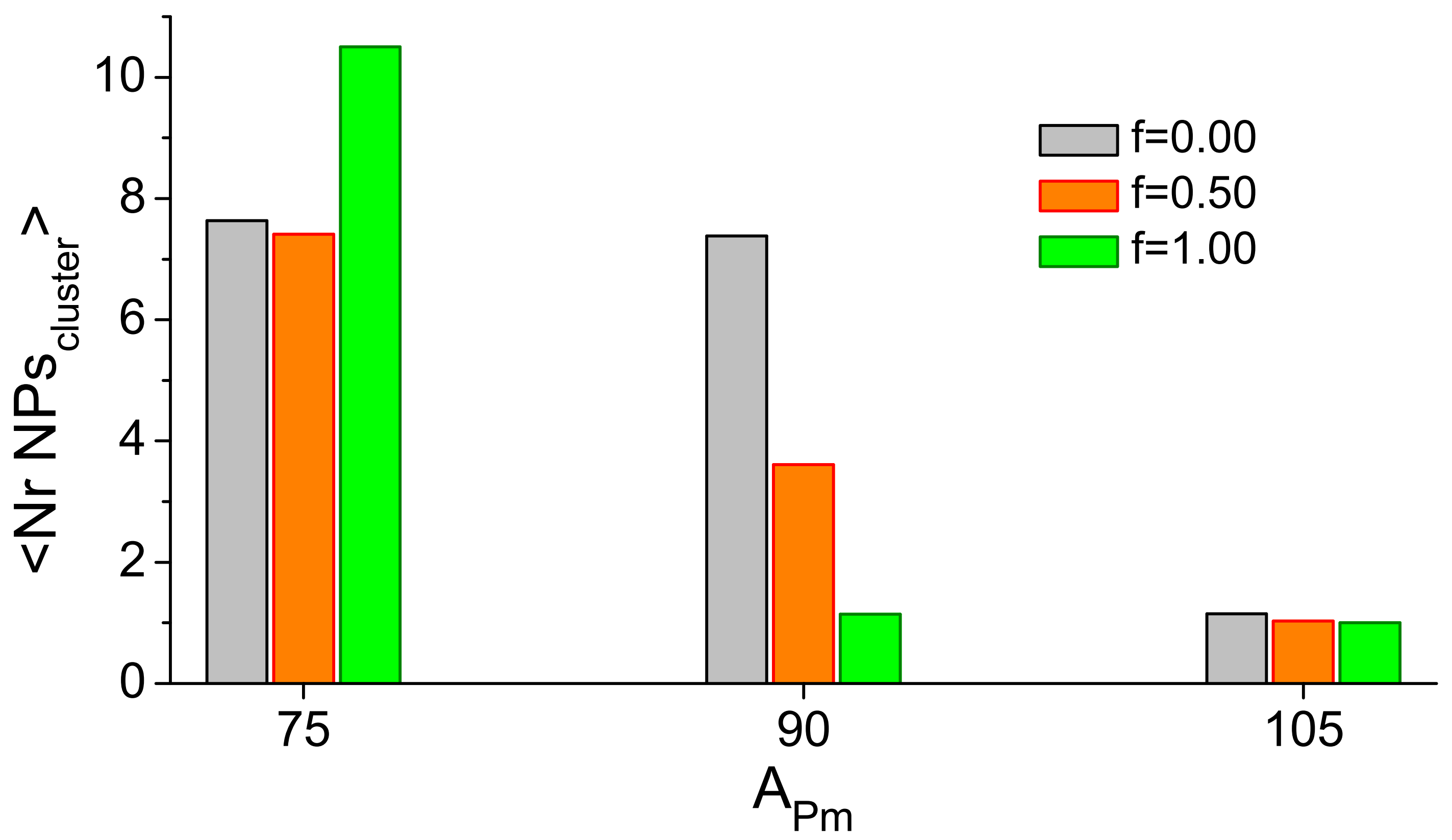



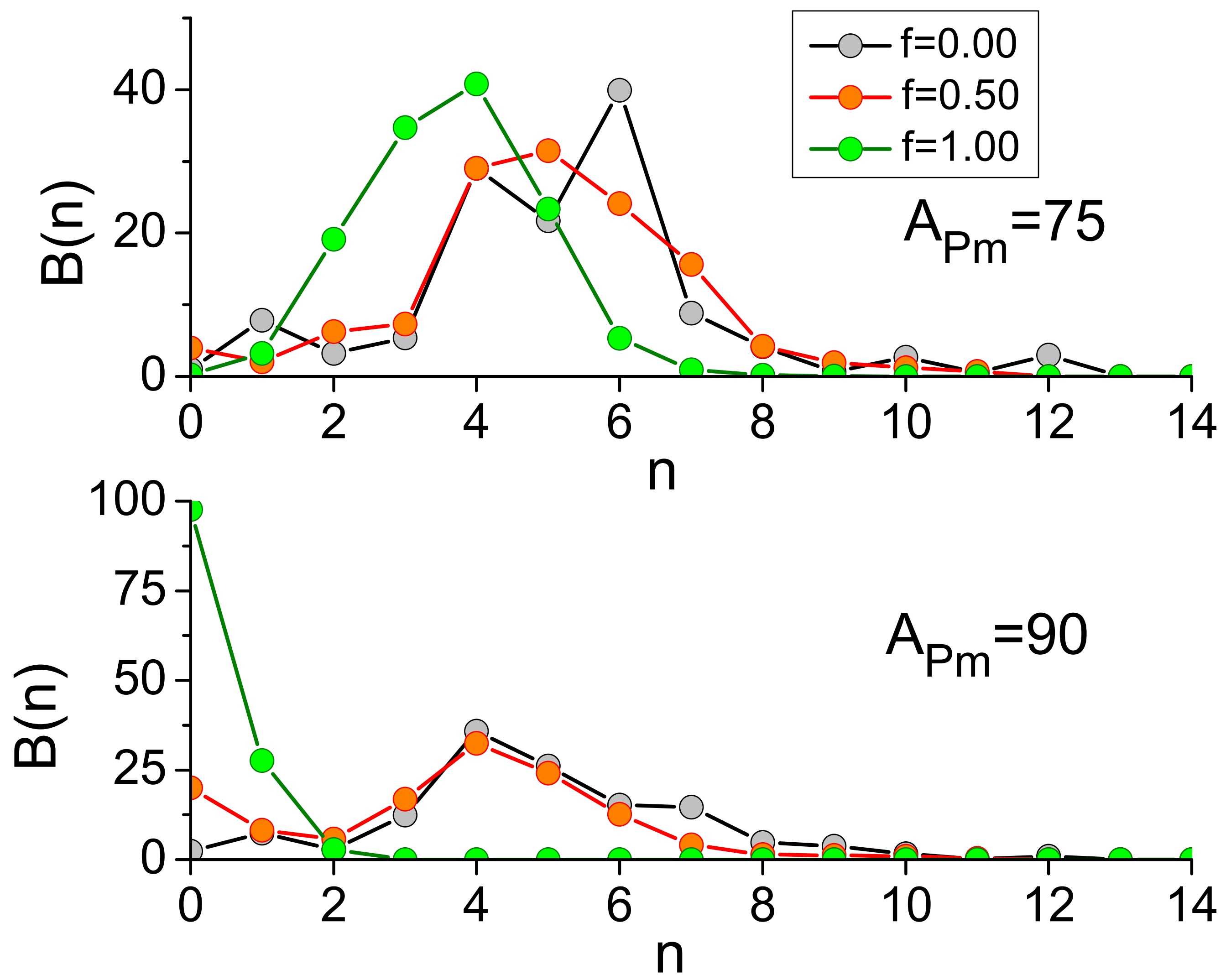